\title{
Pluripotent Stem Cells: Cancer Study, Therapy, and Vaccination
}

\author{
Mojgan Barati ${ }^{1,2} \cdot$ Maryam Akhondi $^{2} \cdot$ Narges Sabahi Mousavi $^{2} \cdot$ Newsha Haghparast $^{2} \cdot$ Asma Ghodsi $^{2}$. \\ Hossein Baharvand ${ }^{1,2} \cdot$ Marzieh Ebrahimi $^{2} \cdot$ Seyedeh-Nafiseh Hassani ${ }^{2,3}$ (D)
}

Accepted: 2 June 2021 / Published online: 11 June 2021

(c) The Author(s), under exclusive licence to Springer Science+Business Media, LLC, part of Springer Nature 2021

\begin{abstract}
Introduction Pluripotent stem cells (PSCs) are promising tools for modern regenerative medicine applications because of their stemness properties, which include unlimited self-renewal and the ability to differentiate into all cell types in the body. Evidence suggests that a rare population of cells within a tumor, termed cancer stem cells (CSCs), exhibit stemness and phenotypic plasticity properties that are primarily responsible for resistance to chemotherapy, radiotherapy, metastasis, cancer development, and tumor relapse. Different therapeutic approaches that target CSCs have been developed for tumor eradication.

Results and Discussion In this review, we first provide an overview of different viewpoints about the origin of CSCs. Particular attention has been paid to views believe that CSCs are probably appeared through dysregulation of very small embryoniclike stem cells (VSELs) which reside in various tissues as the main candidate for tissue-specific stem cells. The expression of pluripotency markers in these two types of cells can strengthen the validity of this theory. In this regard, we discuss the common properties of CSCs and PSCs, and highlight the potential of PSCs in cancer studies, therapeutic applications, as well as educating the immune system against CSCs.

Conclusion In conclusion, the resemblance of CSCs to PSCs can provide an appropriate source of CSC-specific antigens through cultivation of PSCs which brings to light promising ideas for prophylactic and therapeutic cancer vaccine development.
\end{abstract}

Keywords Cancer stem cells $\cdot$ Embryonic stem cells $\cdot$ Pluripotency $\cdot$ Cancer vaccine

Mojgan Barati and Maryam Akhondi authors contributed equally to this work.

Seyedeh-Nafiseh Hassani snafiseh.hassani@royaninstitute.org;

sn.hassani@royan-rc.ac.ir

Marzieh Ebrahimi

m.ebrahimi@royan-rc.ac.ir

1 Department of Developmental Biology, School of Basic Sciences and Advanced Technologies in Biology, University of Science and Culture, Tehran, Iran

2 Department of Stem Cells and Developmental Biology, Cell Science Research Center, Royan Institute for Stem Cell Biology and Technology, ACECR, Tehran, Iran

3 Advanced Therapy Medicinal Product Technology Development Center (ATMP-TDC), Cell Science Research Center, Royan Institute for Stem Cell Biology and Technology, ACECR, Tehran, Iran

\section{Introduction}

Cancer is a major worldwide health issue and leading cause of death. It is estimated that the worldwide cancer rate may surpass 29 million cases annually with approximately 1 out of every 6 deaths in 2040 [1]. Despite outstanding progress in many therapeutic approaches, various factors such as the failure of current treatments, drug resistance [2], delayed diagnosis [3] and absence of a strong immune system in affected patients [4] have led to the lack of a definitive cancer treatment. Most importantly, metastasis post-treatment and recurrence of tumors after resection are the main causes of cancer death [5-7]. Therefore, therapeutic strategies, which can treat or prevent tumors from metastasis and recurrence could be an outstanding achievement in cancer therapy.

Currently, the role of cancer stem cells (CSCs) in the propagation of malignant tumors and resistance to therapy has become more accentuated. CSCs comprise a group of cells that can form transplantable tumors with the same 
cellular heterogeneity that exists in their tumor of origin [8]. Beside the ability both to self-renew and to differentiate into several heterogeneous cells with less potency, major disorders related to malignancy such as plasticity, drug resistance, epithelial to mesenchymal transition (EMT) and metastasis, and tumor recurrence are attributed to the existence of CSCs. Many attempts have been made to isolate and characterize CSCs to enable further diagnosis and treatment; however, the lack of specific markers for cellular recognition is a serious challenge in dealing with CSCs [9]. There are several questions that should be answered about CSCs and understanding the origin of these cells may help define a useful model for further studies.

Pluripotent stem cells (PSCs), with an indefinite selfrenewal capacity and the potential to generate all cell types of the body, have many similarities with CSCs. PSCs are usually derived via direct cultivation of early embryos (embryonic stem cells [ESCs]) or from reprogramming of somatic cells (induced PSCs [iPSCs]) [10, 11]. These cells have been used as promising sources for cell based therapies in clinical trials of spinal cord injuries, diabetes and agerelated macular degeneration (AMD) treatment (reviewed in [12]). However, PSC-based cancer therapy studies are mainly restricted to derivation of functional cancer fighting cells that include PSC-derived dendritic cells (DCs) [13], lymphocytes [14], and transplantable cells like hematopoietic stem cells (HSCs) [15]. The results of some studies have shown that exposure of different types of cancer cells to the PSC microenvironment could inhibit Nodal expression and suppress the malignant characteristics of these cells [16]. The importance of these PSC-based research priorities signifies a novel vaccination strategy against CSCs that possibly could induce long-term memory against a broad-spectrum of tumors [17].

In this review, we discuss the existing theories that concern the origins of cancer or, more specifically, those of CSCs, their resemblance to PSCs and application of PSCs against tumors as a novel cancer therapy approach.

\section{Cancer Stem Cells (CSCs) as Stem Cell Malignancies: Origin of CSCs}

Growing evidence support the idea of representing cancer as a stem cell malignancy and the introduction of CSCs as being responsible for metastasis and tumor recurrence [18, 19]. Cell-cell fusion, horizontal gene transfer, genetic instability and the cell microenvironment are proposed mechanisms for these malignancies [20]. Accordingly, there are various assumptions about the origin of CSCs that include disrupted tissue-resident stem cells such as specific adult stem cells and their precursors, dedifferentiated somatic cells, and improperly activated residual embryonic cells in adult tissues.

\section{Residual Embryonic Cells}

The presence of cells with a semi-embryonic morphology in cancerous biopsies was initially reported in the nineteenth century. This finding ultimately led to the formation of a hypothesis entitled "embryonic rest of cancer origin" developed by Rudolf Virchow and his student Julius Cohnheim, which suggests that the remnants of embryonic cells persist in adult tissues and remain silent until adulthood [21]. In certain conditions, however, these cells can be activated by the influence of internal and external factors and they play a role in cancer development [22]. The close relation between embryonic and cancer cells was reported in cancers such as nephroblastomas that originate from germ cell tumors [23]. Researchers also identified some cells with embryonic morphology nominated as "very small embryonic-like stem cells" (VSELs). Possibly, during early embryogenesis when primordial germ cells (PGCs) originate from the proximal epiblast, a group of PGCs seats in other tissues where they remain silent. Therefore, it was hypothesized that migrating PGCs from the primary epiblast may be the origin of VSELs [24]. There is a hypothesis that places these cells at the top of the stem cell hierarchy in bone marrow, which results in the production of HSCs, mesenchymal stem cells (MSCs), endothelial progenitor cells, and production of tissue-committed stem cells (TCSCs) in other tissues (Fig. 1) [25]. Furthermore, it is suggested that VSELs are a type of migratory stem cells that are released from bone marrow into the bloodstream during stressful conditions and participate to regenerate damaged tissues [26]. These cells could be found in bone marrow, brain, pancreas, thymus and intestinal epithelium, and ovarian and testis surface epithelium in human and rodents [25, 27]. Improperly, fusion of normal cell with VSEL as a circulating stem cell provides the possibility of formation cancer initiating cell or CSCs in solid tumors [26]. VSELs are also isolated from human ESCs' culture using stem-cell-related markers (CD133, SSEA-4) and germinal lineage markers (DDX4/VASA, PRDM14) [28]. These cells possess many characteristics of pluripotent cells; for example, a prototype of undifferentiated morphology with high chromatin content in the nucleus and high nucleus to cytoplasm ratio; presence of bivalent domains in chromatin; pluripotency marker expressions such as Oct4, NANOG, and SSEA1 (murine) or SSEA4 (humans); and the ability to form spheres and in vitro differentiation into derivatives of the three germ layers [29, 30]. Moreover, like ESCs, these cells have elevated telomerase activity and lack MHC class I expression on their surfaces [29, 31-33]. These also express germ cell markers such as Stella and Blimp1, as well as epiblast markers Gbx2, Fgf5 and Nodal [24]. 


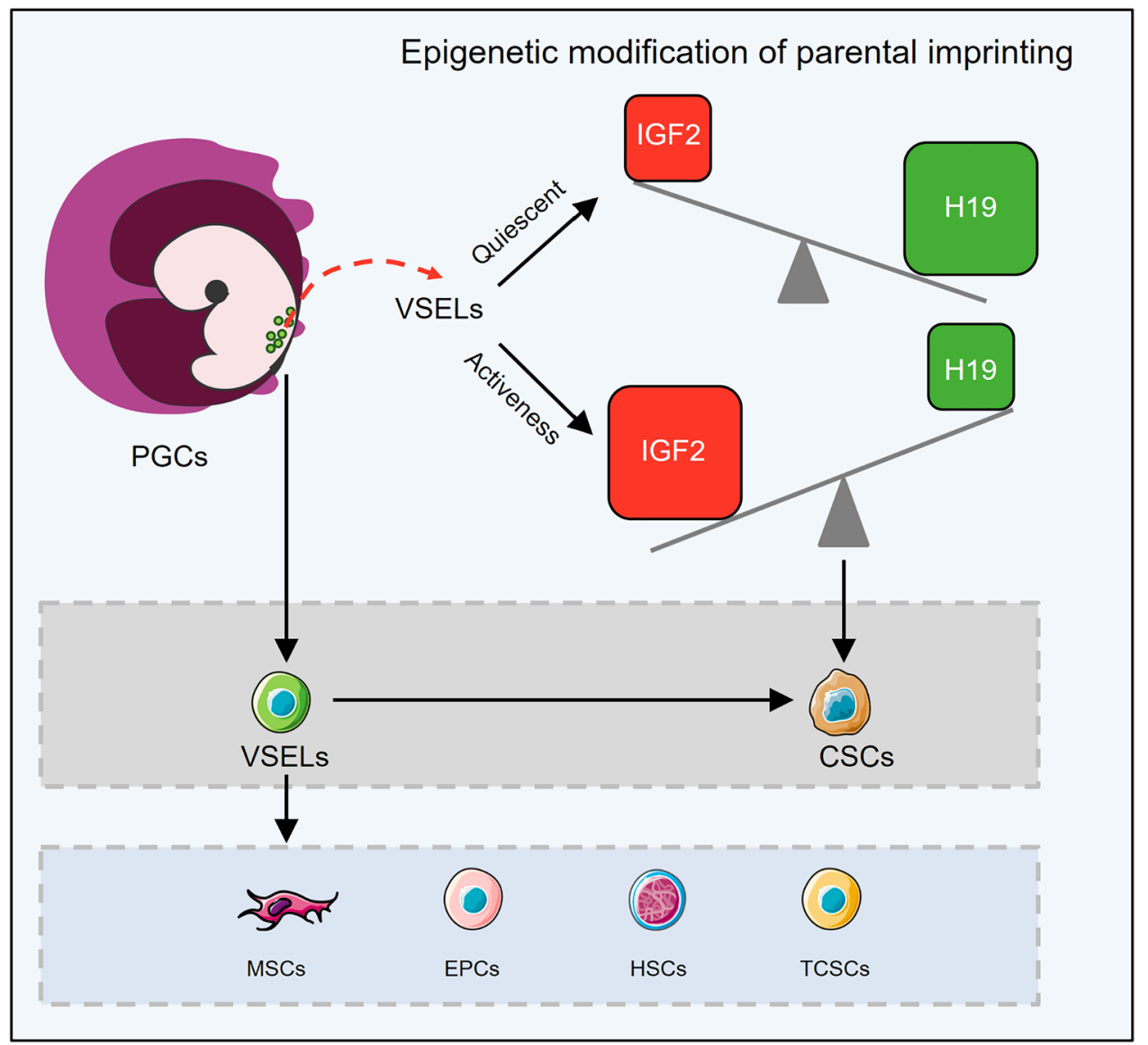

Fig. 1 Very small embryonic-like stem cells (VSELs) support the embryonic rest theory. Primordial germ cells (PGCs) and VSELs are connected by a similar molecular signature. VSELs are present in variety of tissues and are thought to be a source of mesenchymal stem cells (MSCs), hematopoietic stem cell (HSCs), and endothelial progenitor cells (EPCs) in bone marrow as well as tissue-specific stem/ progenitor cells in other tissues. Microenvironment condition, mutations, and epigenetic modifications can change the fate of VSELs into cancer initiating cells or cancer stem cells (CSCs). In the quiescent

To have a historical look at the development of VSELs, it should be referred to the mechanism of PGCs migration in the gastrulating embryo. At the onset of PGC migration, the complete erasure of imprinting marks from DNA of these cells results in downregulation of insulin-like growth factor 2 (IGF2) and upregulation of the tumor suppressor gene H19, which promote and inhibit growth, respectively [34]. Indeed, parental allele-specific expression of $\mathrm{Igf} 2 / \mathrm{H} 19$ genes is controlled by differentially methylated regions (DMRs) and imprinting control regions (ICRs) which its regulation relies on DNA methylation. Normally, growth factor gene $I g f 2$ is expressed from the paternal allele and tumor suppressor gene $\mathrm{H} 19$ is expressed from the maternal allele. These genes are neighboring genes, and both respond to the same enhancers located at H19's distal end. Briefly, the monoallelic expression of $I g f 2$ is mediated by allele-specific DNA state, VSELs highly express growth-repressive imprinted gene transcripts such as $\mathrm{H} 19$ and show the downregulation of growth-promoting ones like IGF2. However, in activated VSELs, there is higher expression of IGF2 compared to H19, which ultimately leads to uncontrolled cell proliferation and can promote cancer formation. The cartoon in all figures of this article was created using Servier Medical Art templates (https://smart.servier.com) licensed under a Creative Commons License (https://creativecommons.org/licenses/by/3.0/)

methylation at CTCF-binding sites in ICR, located immediately upstream of the $H 19$ gene. Actually, CTCF (known as CCCTC-binding factor) mediates the insulation at the H19Igf2 imprinted domain [35]. The methylated paternal ICR inhibits CTCF protein binding, which allows the Igf2 promoter to interact with the enhancers and be transcribed. Unmethylated maternal ICR binds to CTCF and prevents the $I g f 2$ promoter from interacting with the enhancers. As a result, only $\mathrm{H} 19$ is transcribed on the maternal chromosome. However, in the late migratory PGCs, and similarly in developing VSELs, the complete erasure of imprinting marks from DNA results in down-regulation of $I g f 2$ and up-regulation of $\mathrm{H} 19$ which led to the prevention of uncontrolled proliferation in these cells and explain the quiescent state of VSELs in adult tissues (Fig. 1) [34]. Improperly, if VSELs in adult testes affected by endocrine disruption and 
alteration of epigenetic modifications in imprinted genes, they may initiate self-renewal and promote testicular cancer [36]. Furthermore reduced sperm count and infertility can occur as a result of increased VSEL proliferation and impaired differentiation [37]. According to Ratajczak and his colleagues, VSELs can convert to CSCs and promote the formation of teratomas and teratocarcinomas, germinal tumors, or pediatric sarcomas if they achieve relevant mutations [26]. The presence of VSELs in ovarian teratoma and testicular cancer suggests these cells as tumor-initiating stem cells. In these tumors, VSELs express both pluripotency and germinal lineage markers and suggests to play important role in tumor invasion through the activation of EMT [38]. On the other hand, like VSELs, the expression of cancer testis antigens (CTAs; for example: MageB3, Ssbx2, and BORIS) and epiblast/germline-related cells are seen in some tumors $[26,38]$. It's worth noting that identifying these small putative NANOG-, SOX2-, and SSEA-4-positive cells that are responsible for tumor formation in ovarian cancer is crucial from a clinical point of view as the potential therapeutic targets [39].

\section{Tissue-specific Stem/Progenitor Cells}

Tissue-specific stem/progenitor cells, also known as adult stem cells, are a rare cell population that have self-renewal and multi-lineage differentiation abilities. These cells are present in various tissues such as the intestines [40], breasts [41], lungs [42], ovaries [43] and prostate [44] and they function to repair or maintain these tissues. In a "classical model", it has been assumed that the asymmetric mitotic division invariably creates one new stem cell and one daughter cell in each niche space of the adult tissues. However, in a "neutral competition model" it is supposed that each of the daughter cells, and even fully differentiated cells, can re-enter the niche and dedifferentiate to replace lost stem cells. The evidence to support this claim is that, with some rare exceptions such as muscle satellite cells or hair-follicle stem cells, the finding of such tissue-specific stem cells in most tissues of the body is almost impossible [45].

On the other hand, tissue-specific stem/progenitor cells are also considered as a primary suspect of cancer onset. However, definitions of these cells described in some studies, for example the assuming of a quiescent state of these cells during the lifetime of their host [46] and their activation under adverse conditions such as tissue damage that may lead to the conversion of these cells to CSCs is more in line with the definition that applies to the VSELs.

It is generally believed that the proliferation of tissuespecific stem/progenitor cells is precisely under control while they also are prompt enough to take action in confronting any awakening signal [47]. Furthermore, these cells can maintain their homeostasis and survival during differentiation by controlling the metabolic process of autophagy [48]. By receiving different signals from their surrounding cells and the microenvironment, tissue-specific stem/progenitor cells can quit quiescence and regain selfrenewal, proliferation, and the ability to differentiate into different cell types, which enables them to maintain an effective role in regeneration [49]. However, it cannot be ignored the accumulation of genetic and epigenetic mutations that specifically be caused by aging may exert adverse effects on the accuracy of self-renewal and cell survival pathways of these cells [50-52]. Moreover, it is also possible that issue-specific stem/progenitor cells may turn into cancer initiating cells by fusion with abnormal cells or by absorbing damaging cellular material from cells trapped by phagocytosis and cause alterations in their normal mechanisms [53]. It is likely that processes such as autophagy can apply changes to the stem cell cellular microenvironment, homeostasis, and maintenance, and affect adult stem cell survival by inducing apoptosis or a cancerous state (Fig. 2) [54-57].

On the other hand, CSCs can generate progenitors that have self-renewal ability and less stemness capability, which is similar to multipotent tissue-specific stem/progenitor cells [46, 58]. In addition, signaling networks, like the Wnt and Notch pathways, are common between CSCs and multipotent tissue-specific stem/progenitor cells [59, 60], and signal transduction occurs through some of the same surface receptors such as CD24 [61], CD44 [62], CD133 [63, 64], and CD49f $[65,66]$. This data suggests a close relationship between these two cell types [42, 44, 67].

\section{Stem/Progenitor Cells Produced by Reprogramming}

In 2006, Takahashi and Yamanaka established a major breakthrough with the generation of iPSCs by reprogramming differentiated somatic cells into the pluripotent state by induction of four transcription factors (TFs) - Oct4 (Pou5f1), Sox2, c-Myc, and KLF4 [11, 68]. Since most of these TFs are oncogenes [69], genetic and epigenetic alterations in differentiated somatic cells have been hypothesized to activate dedifferentiation processes in adult cells to convert them to CSCs [70]. In this scenario, the expression of differentiation-specific genes in somatic cells is gradually downregulated and upregulation of stemness-related genes occurs, which leads to the establishment of a pluripotencylike state. This transformation has been observed in basallike human mammary epithelial cells [71] and differentiated airway epithelial cells [72]. Similarly, in intestinal tumors, activation of NF- $\mathrm{KB}$ in response to inflammatory pathways leads to activation of Wnt signaling and consequently results in reprogramming of non-stem cell intestinal epithelial cells (IEC) into a pluripotent state [73]. Proto-oncogene activating 


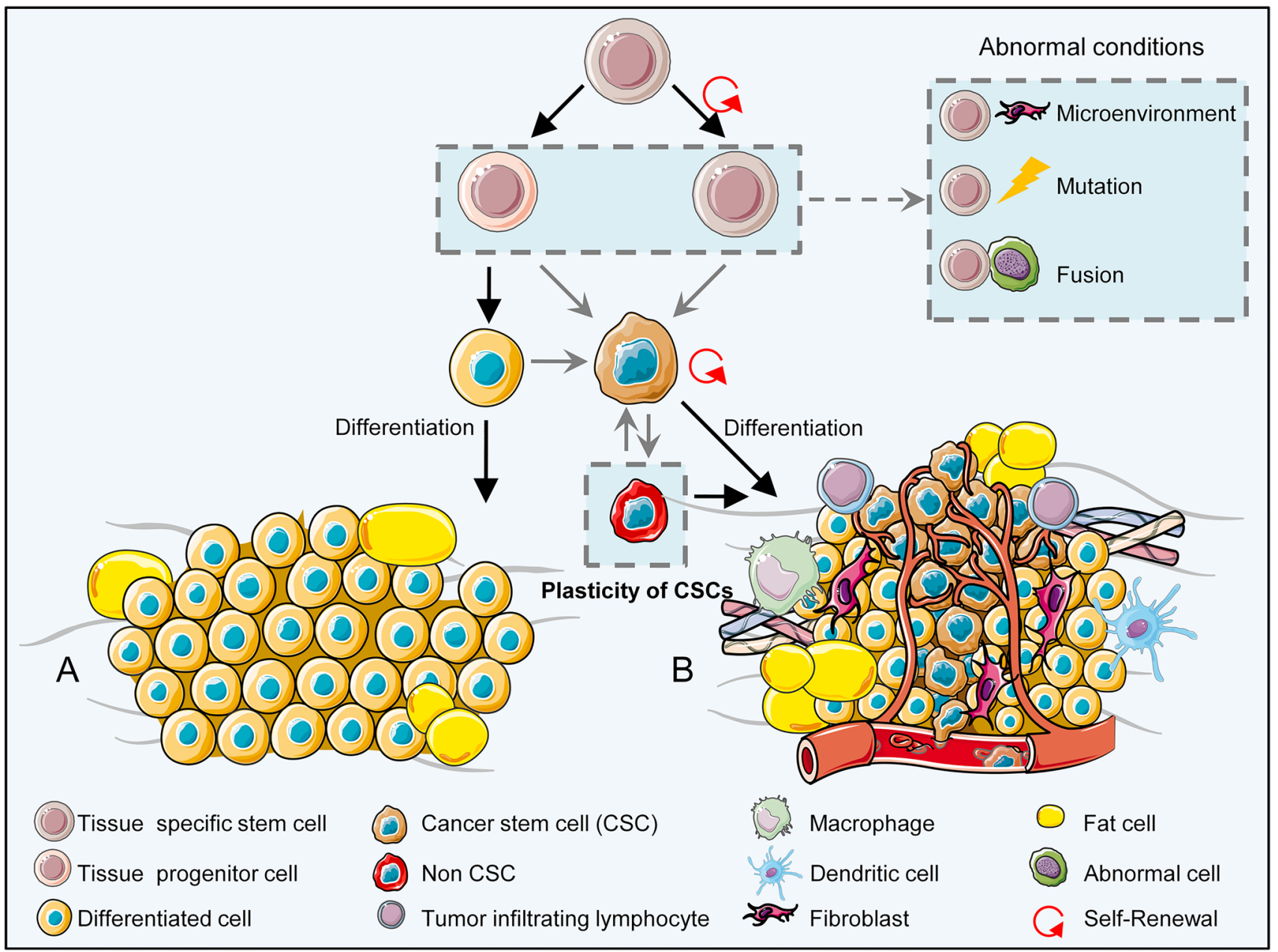

Fig. 2 Schematic illustration of transformation of specific tissue stem cells into cancer stem cells (CSCs). A) Tissue-specific stem cells normally program into precursor cells that can differentiate into specified tissue cells. B) Cancer-derived external (i.e., microenvironment) or internal (genetic or epigenetic) factors or fusion with an abnormal

mutations such as KRas and its downstream target c-Myc are among the other dynamic ways that transform cells into cancer stem-like cells. Interestingly, KRas activating mutations have been detected in over $90 \%$ of pancreatic cancers and is one of the key mutations in various cancers [74-76].

The tumor microenvironment is another important factor that may contribute to transformation of differentiated cells into stem-like cells. In colorectal tumors, cancer-associated myofibroblasts can induce dedifferentiation pathways by secreting hepatocyte growth factor (HGF) that consequently activates Wnt signaling, which enhances stemness properties in differentiated cells [77]. T-cells and macrophages can direct dedifferentiation of differentiated cells through the secretion of tumor necrosis factor alpha (TNF- $\alpha)$ in the induced inflammatory microenvironment in melanoma. On the other hand, it has been assumed that these microenvironments induce plasticity in tumor cells that lead to changes cell can cause tissue specific stem cells or their precursor derivatives to revert into a cancerous state. Immune cells are suppressed by cancer cells in the affected area. Other normal resident cells, such as fibroblasts, may change in favor of the tumor and create a new microenvironment

in certain surface markers and the resistance of the tumor cells to an anti-cancer immune response [78]. In general, the tumor microenvironment increases cellular plasticity, which induces various cell types at various differentiation levels to transform into CSCs (Fig. 3).

\section{Comparisons between Pluripotent Stem Cells (PSCs) and Cancer Stem Cells (CSCs): Key Factors}

Regardless of the cell of origin, the stemness characteristics and the resemblance of CSCs to early developmental cells is significant in the stem cell theory of cancer (Fig. 4). In many ways cancer cells imitate the early developmental pathways at the biological and molecular levels. The activation of initial developmental signaling pathways [79] and the TF expressions 
Fig. 3 Adult stem cell reprogramming. Tissue adult stem cells are usually unipotent; however, under microenvironment-induced transformation, the activation of pluripotencyrelated signaling pathways like Wnt and transcription factors (TFs), such as Oct4, lead to their reprogramming into a pluripotent state The newly formed cells go through genetic and epigenetic alterations that favor cancer stem cell (CSC) formation

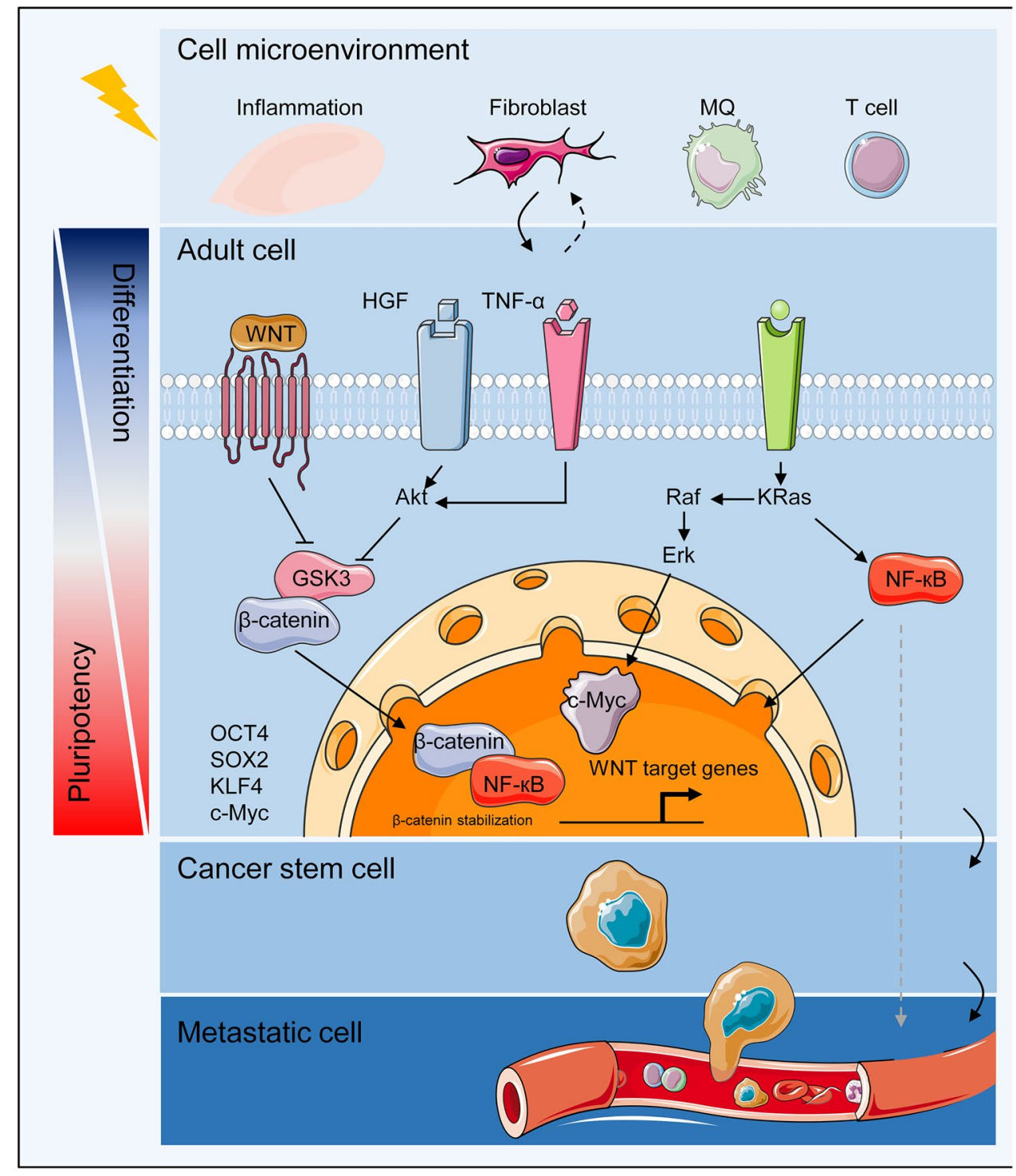

associated with pluripotency in both CSCs and PSCs [80] (Table 1). Moreover, the similarities in protein content [79] and metabolism [81] can show the proximity of CSCs and PSCs.

\section{Key Transcription Factors (TFs)}

The induction of the main pluripotency-associated TFs Oct4, Sox2, KLF4 and NANOG results in induction of pluripotency in terminally differentiated cells into iPSCs. These TFs are considered to be key controllers of stemness in CSCs [82]. Oct4 plays an important role in germ cell tumor formation and is a diagnostic factor in these tumors. Post-translational modifications of Oct4 increase its stability in breast CSCs [83]. Oct4 is considered to be a renal CSC marker along with high expression of NANOG, and it is responsible for maintenance of CSCs, which negatively affects survival [84]. Oct4 expression increases stemness, drug resistance and migration of pancreatic CSCs (PCSCs) [85]. Similar to Oct4, NANOG plays an important role in maintaining malignant stem cell populations and CSCs of glioblastoma, liver, colon and prostate cancers [86-89]. Sox 2 expression can be seen in squamous cell carcinoma of the esophagus [90] and small cell lung carcinoma [91], and its expression is necessary in maintenance of the CSC microenvironment in glioblastoma and breast cancer [92].

On the other hand, KLF4, is a double-edged sword in cancer development. Although this TF promotes malignancies in bladder urothelial carcinoma and osteosarcoma [93, 94], its inhibitory role in colorectal cancer [95], neuroblastoma [96], and nasopharyngeal sarcoma [97] has been reported. KLF4 interaction with c-Myc, a proto-oncogene, and its dissuasive effect on p53, a tumor suppressor, results in an increased proliferation and enhanced reprogramming that can be seen in p53 deficiency. Lack of efficient p53 
Fig. 4 Yin-yang of pluripotency. While stemness and pluripotency in the early embryo are crucial factors in creating a new being, the activation of pluripotency in some cells, such as very small embryonic-like stem cells (VSELs) and tissue-specific stem cells in adult tissues leads to formation of cancerous cells and cancer stem cells (CSCs)

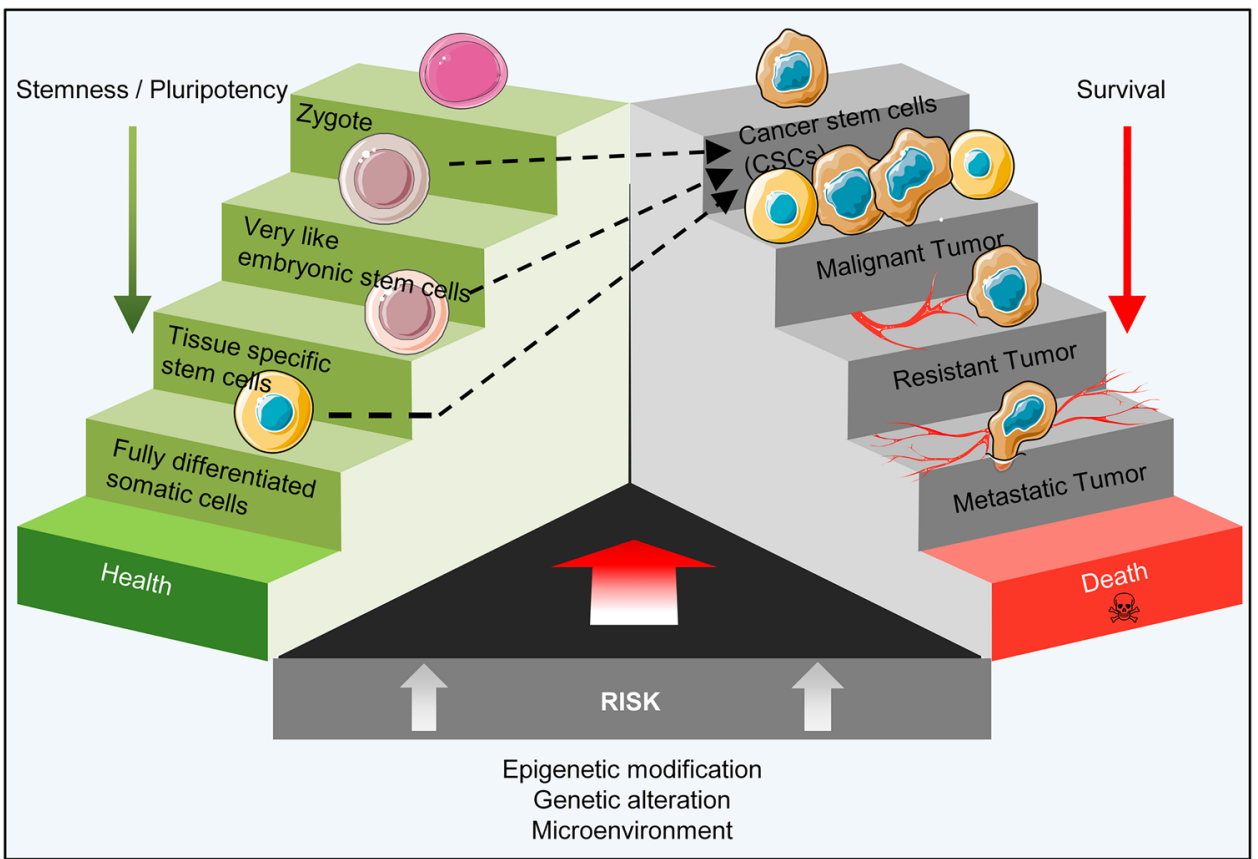

expression can result in tumor formation in the adult stem cell populations, and diminish their differentiation and trigger malignant features in iPSCs [98]. KLF4 also triggers induction of telomerization activity in cancer cells, which is similar to ESCs [99]. C-Myc augments cell proliferation in many human malignancies and plays a role in reprogramming of fibroblasts to iPSCs [100]. This factor also affects the enzymes involved in transcription and can increase total transcription levels [101]. Together, these factors form a network of interactions that have two sides-one promotes malignancy and the other is responsible for the pluripotent state of cells. The balance between these two defines the normal cell state.

Table 1 Pluripotency-related TF in PSCs and CSCs

\begin{tabular}{|c|c|c|c|}
\hline Pluripotency marker & Role in cancer development & Type of Cancer & References \\
\hline \multirow[t]{3}{*}{ OCT4 } & \multirow[t]{3}{*}{ Stemness, EMT, Metastasis and drug resistance } & Breast cancer & {$[168-170]$} \\
\hline & & Pancreatic cancer & {$[171]$} \\
\hline & & Liver cancer & {$[172]$} \\
\hline \multirow[t]{10}{*}{ SOX2 } & \multirow[t]{7}{*}{ Stemness, EMT, Metastasis and drug resistance } & Squamous cell carcinoma & {$[90,173]$} \\
\hline & & Small cell lung carcinoma & [91] \\
\hline & & Non- Small cell lung carcinoma & [174] \\
\hline & & Glioblastoma & {$[175]$} \\
\hline & & Colorectal cancer & {$[176]$} \\
\hline & & Bladder Urothelial carcinoma & [93] \\
\hline & & Osteosarcoma & [94] \\
\hline & \multirow[t]{3}{*}{ Tumor suppressor } & Colorectal cancer & [95] \\
\hline & & Neuroblastoma, & [96] \\
\hline & & Nasopharyngeal sarcoma & {$[97,177]$} \\
\hline \multirow[t]{5}{*}{ NANOG } & \multirow[t]{5}{*}{ Stemness, EMT, Metastasis and drug resistance } & Glioblastoma & [86] \\
\hline & & Liver & {$[87,178]$} \\
\hline & & Colorectal cancer & {$[88]$} \\
\hline & & Prostate cancer & {$[89,179]$} \\
\hline & & Gastric cancer & [180] \\
\hline
\end{tabular}


Table 2 Common regulatory signaling cascades in PSCs and CSCs

\begin{tabular}{|c|c|c|c|c|}
\hline Signaling pathway & Targets & Role in cancer development & Cancer type & References \\
\hline \multirow[t]{11}{*}{ Notch } & \multirow[t]{8}{*}{$\begin{array}{l}\text { AKT, NF-kB, p21, p27 and } \\
\text { CyclinD1 }\end{array}$} & \multirow{8}{*}{$\begin{array}{l}\text { Pluripotency maintenance, } \\
\text { cell fate determination and } \\
\text { proliferation and apoptosis } \\
\text { regulation }\end{array}$} & $\begin{array}{l}\text { Pancreatic Ductal Adenocarci- } \\
\text { noma (PDAC) }\end{array}$ & {$[117,181,182]$} \\
\hline & & & Melanomas & [107] \\
\hline & & & Medulloblastoma & {$[183]$} \\
\hline & & & Ovarian cancer & [109] \\
\hline & & & Breast cancer & [110] \\
\hline & & & Prostate cancer & [184] \\
\hline & & & Hepatocellular Carcinoma & {$[185]$} \\
\hline & & & $\begin{array}{l}\text { Non-Small Cell Lung Cancer } \\
\text { (NSCLC) }\end{array}$ & [186] \\
\hline & \multirow{3}{*}{$\begin{array}{l}\text { Cyclin A, Cyclin D1, Cyclin E, } \\
\text { CDK2, Retinoblastoma pro- } \\
\text { tein, HES-1, P21, P63 and P53 }\end{array}$} & \multirow[t]{3}{*}{ Tumor suppressor } & $\begin{array}{l}\text { Cutaneous Squamous Cell } \\
\text { Carcinoma (CSCC) }\end{array}$ & [187-189] \\
\hline & & & $\begin{array}{l}\text { head and neck squamous cell } \\
\text { carcinoma (HNSCC) }\end{array}$ & {$[190]$} \\
\hline & & & Small Cell Lung Cancer (SCLC) & {$[191,192]$} \\
\hline \multirow[t]{6}{*}{ WNT } & \multirow[t]{6}{*}{$\beta$-catenin and STAT3 } & \multirow{6}{*}{$\begin{array}{l}\text { Pluripotency maintenance,Stem } \\
\text { cell self-renewal and differen- } \\
\text { tiation and EMT }\end{array}$} & Colorectal Carcinoma & [118] \\
\hline & & & Leukemia & [114] \\
\hline & & & Melanoma & [119] \\
\hline & & & Liver cancer & {$[120]$} \\
\hline & & & Prostate cancer & {$[121]$} \\
\hline & & & Breast cancer & [122] \\
\hline \multirow[t]{7}{*}{ Hedgehog (HH) } & \multirow[t]{7}{*}{ GLI families } & \multirow{7}{*}{$\begin{array}{l}\text { Pluripotency maintenance and } \\
\text { Stem cell self-renewal }\end{array}$} & Basal Cell Carcinoma & {$[193,194]$} \\
\hline & & & Medulloblastoma & {$[123,125,193,195]$} \\
\hline & & & Colon cancer & [196] \\
\hline & & & Breast cancer & [197] \\
\hline & & & $\begin{array}{l}\text { Chronic Myelogenous Leukemia } \\
\text { (CML) }\end{array}$ & [198] \\
\hline & & & $\begin{array}{l}\text { T- cell Acute Lymphoblastic } \\
\text { Leukemia (T-ALL) }\end{array}$ & [199] \\
\hline & & & $\begin{array}{l}\text { pancreatic Ductal Adenocarci- } \\
\text { noma (PDA) }\end{array}$ & {$[200]$} \\
\hline
\end{tabular}

\section{Signaling Pathways}

Self-renewal and proliferation are two characteristics of both CSCs and PSCs; however, unlike PSCs, these mechanisms are not strictly controlled by CSCs. Both cells adjust the self-renewal process through the same signaling pathways (Table 2). However, irregular expressions of key factors in CSCs disrupt their balanced state and make them prone to tumor formation [102].

Notch: During development, the Notch signaling pathway is one of the central pathway that makes contact between adjacent cells; it is necessary for differentiation and determination of the cell fate and homeostasis of adult tissues [59]. The Notch signaling pathway reportedly functions in human ESCs (hESCs); however, it appears to be unnecessary for maintenance of pluripotency in these cells. Conversely, Notch signaling plays an important role in maintenance of the differentiating cell types in hESC cultures [103] such as neural differentiation of hESCs [104], as well as migration and proliferation of retinal pigment epithelial cells [105]. Upon activation by direct cell contact, cleaved Notch intracellular domain (NICD) in conjunction with a complex of proteins affects downstream targets that include AKT, NF-kB, p21, p27 and CyclinD1 [106]. Changes in these targets may result in changes in the cell fate, differentiation and uncontrolled proliferation, and may result in melanomas, medulloblastoma, ovarian and breast cancers [102, 107-110]. As a cell context dependent pathway, Notch signaling may also act as a tumor suppressor [111]. Several therapeutic approaches target components of this pathway. Delta like (DLL-1, DLL2, DLL4) or Jagged (JAG1, JAG2) 
ligand, Notch receptors (Notch1-4) and y-secretase complex have been studied. However, in CSCs, several pathways are interconnected and suggested therapies should cover all features of signaling [60].

Wnt: Wnt is one of the most conserved signaling pathways that is active in a wide variety of organisms and is essential for maintenance and self-renewal of adult stem cells (i.e., HSCs) and PSCs [112-114]. Its first connection to cancer was discovered when Wnt overexpression resulted in tumor formation in mice [115]. Nearly a decade later, mutations in the adenomatous polyposis coli (APC) gene were found to be responsible for hereditary colon cancer syndrome [116]. Normally, in the absence of the Wnt ligand, APC along with Axin and GSK3 $\beta$ form a regulatory complex which degrades $\beta$-catenin. Mutated APC activates Wnt signaling by stabilizing $\beta$-catenin, which results in T-cell factor (TCF) 4 transcriptional activity [117]. Non-canonical Wnt signaling, which is $\beta$-catenin independent, may play a role in cancer malignancy by activating planar cell polarity (PCP) and calcium signaling, and result in cell migration and possible metastasis. However, canonical Wnt signaling has been observed more frequently [116]. Telomerase subunit TERT expression after Wnt activation helps to keep telomerase activity, which also links Wnt to CSCs [99]. Other than colorectal carcinoma (CRC) [118], the role of Wnt signaling in leukemia [114], melanoma [119] liver cancer [120], prostate cancer [121] and triple negative breast cancer [122] has been studied and several drugs against this pathway have entered clinical trials [60].

Hedgehog (Hh): Hh signaling is responsible for cell fate determination, neuro-ectodermal lineage differentiation, and polarity and pattern formation during organogenesis. Hh ligand binding to the PTCH1/2 receptor and Smoothened activation enables GLI families of TFs to target the expressions of several genes [123, 124]. Although this pathway is not a key regulator of hESC self-renewal and maintenance [123], disruptions in the normal activity of downstream targets such as the $P T C H I$ and $S M O$ genes have been discovered in patients with basal cell carcinoma (BCC), medulloblastoma, colon cancer, breast cancer, chronic myelogenous leukemia (CML), acute myelogenous leukemia (AML), and acute lymphoblastic leukemia (ALL) [123, 125]. Interestingly non-canonical Hh signaling (Shh-Ptch-SMO independent) seems to be important in pancreatic ductal adenocarcinoma (PDAC). Several different therapies that use SMO antagonists and PTCH1 inhibitors have shown that cancer cells are resistant to some of the SMO inhibitors [125]. Li et al. recently reported that some SMO inhibitor substitutes could be used successfully against this resistance and present a novel opportunity for treatment of medulloblastoma [126].

\section{Surface Antigens}

Tumor and fetal cells have many surface antigens in common; hence, these markers are called oncofetal antigens and they include carcinoembryonic antigen (CEA), prostate-specific antigen (PSA), cancer-testis antigen (CTA), human placental lactogen (hPL), alpha-fetoprotein (AFP), beta-human chorionic gonadotropin ( $\beta$-hCG) and placental alkaline phosphatase (PLAP) [127-131]. Exposure of mature animals to embryonic materials has been shown to increase tumor resistance and tumor transplant rejection [127]. The similarity between the serum antibodies of a cancer patient to a pregnant woman can be attributed to the similarities between cancer and embryonic antigens [132, 133]. Thus, cancer diagnosis during pregnancy may be difficult because both conditions have elevations in the cancer antigen 15-3 (CA 15-3), squamous cell carcinoma antigen (SCC), CA 125, and AFP markers [134]. Many differentiated tissue antigens lose their expressions after tumor formation, which is called "antigenic simplification" [127]. In return, the tumor cells begin to re-express the embryonic genes/ antigens. More importantly, the immunity against tumors created by embryonic material is both humoral and cellular $[17,135]$. Based on these findings, one therapeutic approach against cancer could be a vaccine based on embryonic/fetal materials.

\section{Developmental Biology: A New Approach to Cancer Treatment}

The aforementioned similarities between fetal and cancer cells, especially malignant ones, show that early developmental programs that are active in ESCs and are responsible for fetal development play a role in cancer formation. As these developmental processes are under strict regulation in an embryo, mimicking those regulations may be effective in finding new therapeutic agents against cancer.

\section{Cancer and Cancer Stem Cell (CSC) Modeling Using Pluripotent Stem Cells (PSCs)}

The discovery of the ability to reprogramming of somatic cells into PSCs has developed the view of almost all aspects of biomedical research, including cancer. Recently, similar to iPSC derivation technology, the reprogramming protocols that utilize key pluripotency-associated TFs have been used to induce pluripotency in cancer cell lines. In these studies, melanoma-R545, CML-KBM7, osteosarcoma cell lines-(SAOS2, HOS, MG63), non-small cell lung cancer lines-(H358, H460, IMR90), and gastrointestinal cancer cell lines (GCC) have been placed through dedifferentiation that resulted in the generation of iPSCs. However, some 
of these cells have karyotype abnormalities and are resistance to anti-cancer drugs [136, 137]. On the other hand, in order to observe the cancer formation process, iPSCs were derived from somatic cells that contained germline mutations and subsequently differentiated into lineages that had several early signs of malignancy. This method was used as a model in Li-Fraumeni syndrome [138], myelodysplastic syndrome [139], PDAC [140], and colorectal cancer [141] to determine the role of genetic mutations responsible for these malignancies.

In the CSC modeling related studies, it was shown that exposure of normal iPSCs to the mouse Lewis lung carcinoma (LLC) cell line microenvironment and extracellular vesicles could turn them into cells with CSC phenotypes that had high tumorigenic and metastatic potentials once transplanted into nude mice $[142,143]$. Besides self-renewal, these CSCs could differentiate into endothelial cells that resulted in tumor angiogenesis both in vitro and in vivo [144]. Additionally, drug testing on prostate CSC-like cells derived from iPSCs by the same protocol indicated that these cells are resistant to chemotherapeutic drugs and sensitive to agents that target their stemness [145]. These findings from cancer and CSC modeling with iPSC technology suggest that these cells can help to determine the signaling pathways and mechanisms of tumorigenesis, metastasis, and angiogenesis, and also act as platforms to study and test drugs against stemness and CSC-like characteristics of different types of cancer.

It is important to note that despite the increasing advances in iPSC production and further lineage differentiation with improved protocols, cancer modeling with iPSCs has not yet made much progress. Technically, this limitation can be attributed to two factors. First, some studies have shown that cancer cells are more resistance to reprogramming than normal cells. This resistance may be due to various cancerrelated mutations in cancer cells as the starting material for reprogramming. The second factor is that the process of creating iPSCs from a cancer cell and further lineage differentiation does not work in the cell culture in exactly the same that it does in cancer. As mentioned before, most likely, the cancer starts from VSELs or tissue-specific stem/progenitor cells. Therefore, a proper modeling of cancer with iPSCs that was close to reality must first be able to create progenitor cells in this simulation instead of directly producing the final differentiated cells. Moreover, most differentiated cells from iPSCs are similar to cells in early development rather than phenotypes in adults [146].

Another challenge that limits the modelling of cancer is that iPSCs inherit their starting cells' genetic memory. Therefore, the variable genetic instabilities of the primary cancer cells are captured and amplified in their iPSCs. It may be resolved by isolation of single cells and clonal propagation in order to establishment of a stable original genome.
There are also other challenges related to iPSCs' production per se, such as epigenetic instability, incomplete reprogramming, and line-to line variation, that are true for modeling of cancer with iPSCs, too [146].

\section{Differentiation Therapy by Induction of the Embryonic Microenvironment}

There is an overlap between signaling pathways that control developmental processes and tumor progression. Thus, the question arises whether or not cancer cells can respond to embryonic messages. Several studies have been designed to answer this question. By using matrix conditioned with hESCs, Postovit and colleagues showed an increase in melan-A (MLANA) expression in human melanoma cells and a reduction in their invasiveness [147]. The inhibitory nature of the conditioned medium of ESCs and secretory factors released by these cells increased the number of CSCs in the G1 phase and with the cell cycle arrest without inducing apoptosis [148]. Investigation of intercellular interactions between breast cancer cells and ESCs in a 3D-engineered tumor model showed that low molecular weight factors released by pluripotent cells suppressed Stat 3 signaling and inhibited cancer cell proliferation and migration [149]. Examination of this microenvironment showed that exposed exosomes were the main factors that affected tumor cells and reduced their invasiveness [150]. Notch signaling is also responsible for the antitumor activity of this specific environment against colorectal cancer [151]. Another important factor in the embryonic microenvironment that regulates cancer cell proliferation and differentiation is Gremlin, a BMP4 antagonist. Inhibition of BMP4 activates senescence in melanoma cells [152]. Various miRNAs have been shown to play roles as tumor inhibitors or as oncogenes in cancer. Melanoma cells exposed to hESCs had increased miR-302 expression. An augmented level of miR302 by Notch 4 inhibition causes Nodal reduction. On the other hand, expression of miR-27b, which is responsible for angiogenesis and progression of melanoma in the early stages, is reduced after exposure to the hESCs microenvironment [153]. These studies show the modulation effects of ESCs and their microenvironment on cancer cells, and indicate the potential impact of this approach on cancer treatment. However, the mechanism of these effects is still not clear.

Furthermore, the effects of the embryonic microenvironment on tumor cell progression and metastasis has been investigated in animal models. In a study by Illmensee and Mintz, injection of terato-carcinoma cells into mouse blastocysts not only inhibited the tumor phenotype of the cells, but the terato-carcinoma cells could participate in the formation of normal tissues. In a chick model, the tumor-suppressing activity of the embryonic microenvironment also inhibited sarcoma formation after injection of Rous sarcoma virus 


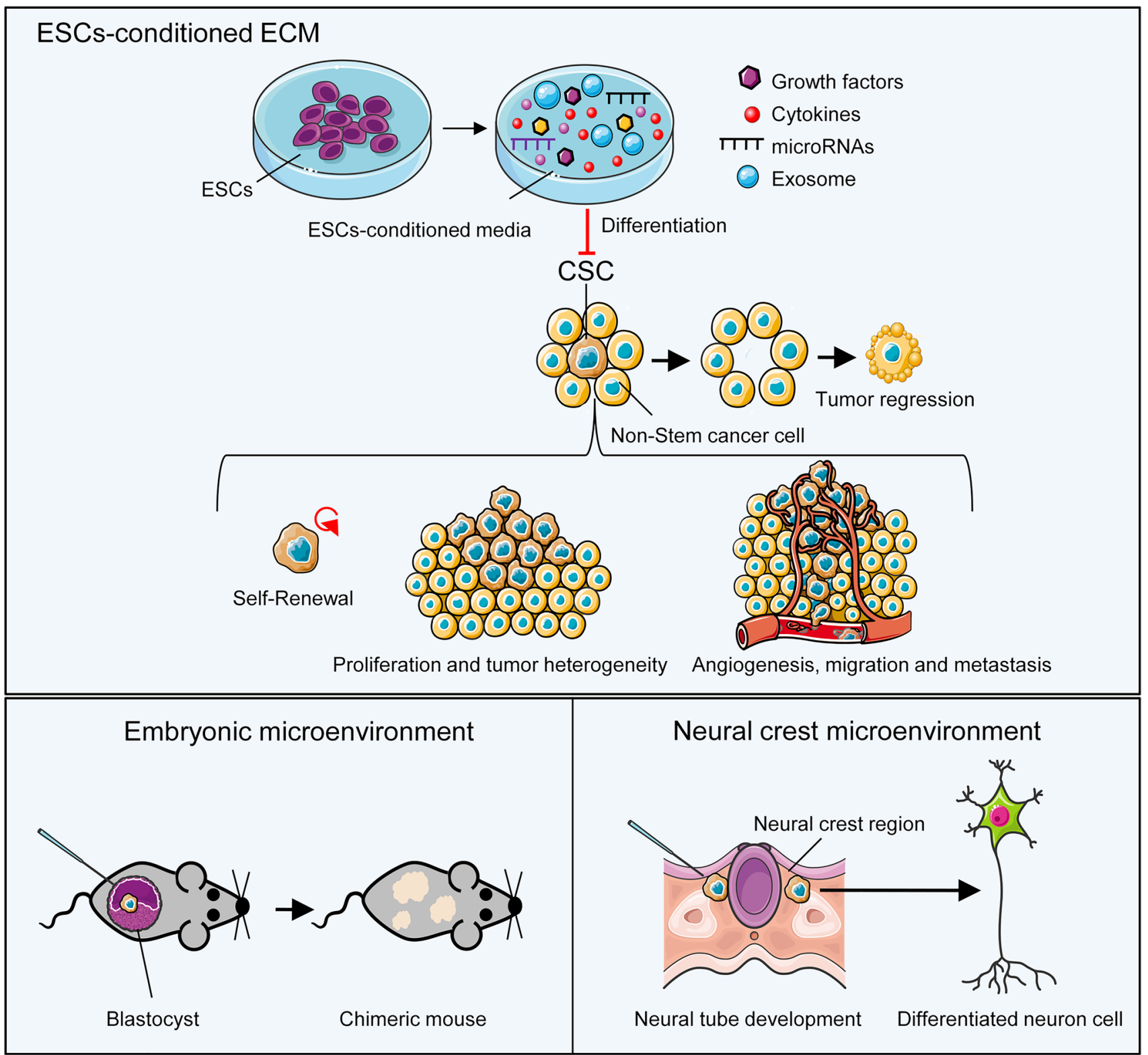

Fig. 5 Differentiation therapy. Communication between the cells and their microenvironment is essential for the maintenance of normal tissues or formation of cancers and their metastases. In cancer cells, there is disrupted regulation of cell differentiation, which leads to the exhibition of a tumor promoting phenotype. The embryonic niche or embryonic stem cell (ESC) conditioned-media can promote

[154]. It has been shown that tumorigenicity of the mousemelanoma cells decreased when the cells were exposed to factors secreted by neural crest region and the cells gained specific markers of differentiated cells such as neural marker TUJ1 [155]. Therefore, embryonic microenvironments appear to provide regulatory factors that are lost in cancer cells during transformation and these factors can be used to restructure embryonic signaling in cancer cells and their microenvironment (Fig. 5). undifferentiated cancer stem cells (CSCs) to become differentiated, which ultimately inhibits tumor growth. After injection into an early embryo, aggressive CSCs learn to "speak the same language" of the embryonic cells and participate in embryo formation instead of tumor growth. The specific niche of the embryo can change the CSC phenotype toward specific cell lineages related to that niche

\section{Using Pluripotent Stem Cells (PSCs) as a Cancer Vaccine}

One of the new potential application of PSCs in cancer research that has been raised in recent years, is the use of PSCs as a global prophylactic cancer vaccine. This idea may seem strange at first; whereas both PSCs and CSCs have common stemness factors, and just as CSCs, PSCs can pose the same threat for body. But, in fact, the 
possibility of large scale cultivation of genetically stable PSCs in vitro, makes available the high amounts of CSCsspecific antigens. In one hand, the conditioned medium of PSCs per se can provide an environment similar to the embryonic environment that promote cancer treatment through differentiation therapy (Fig. 5), and on the other hand, PSCs can be considered as a valuable source to have large amounts of CSCs-specific antigens. Thus far, inactivated PSCs have been used to make prophylactic vaccines against colon [156], lung [17] and ovarian cancer [157] in animal models.

While in the immunosuppressive environment of tumors, cancer cells can suppress the activation of immune cells through various mechanisms, including the recruitment of immunomodulatory MSCs, myeloid-derived suppressor cells (MDSCs), and regulatory T cells (Tregs), the attenuation of MHC class I expression, and the use of the PD-1/PD-L1 axis, cancer vaccines based on cancer-specific antigens can educate the immune system against cancer cells, so that it can encounters and eliminates them. The basic idea behind a cancer vaccine is to find the optimal antigen and vaccine delivery system that is capable of stimulates immune cells. As a result of cancer vaccines, immune responses could be guided selectively toward patients' cancerous cells while preventing their normal cells from immune attack, potentially avoiding side effects. For example, DCs as a professional APC can induce tumor-specific cytotoxicity by processing and presenting tumor antigens to $\mathrm{T}$ lymphocytes. DCs can load with different type of antigens including peptide, RNA and also with whole tumor antigen via tumor lysate or fusion with tumor cells and present numerous antigens to immune effector $\mathrm{CD} 8^{+}$- and $\mathrm{CD} 4^{+}-\mathrm{T}$ cells $[158$, 159]. In addition, induction of immunological memory by DCs overcomes cancer cell plasticity and tumor recurrence [160]. However, DC vaccine therapies require an effective and adequate number of DCs to produce the desired results while in most patients, functional DC is difficult to achieve

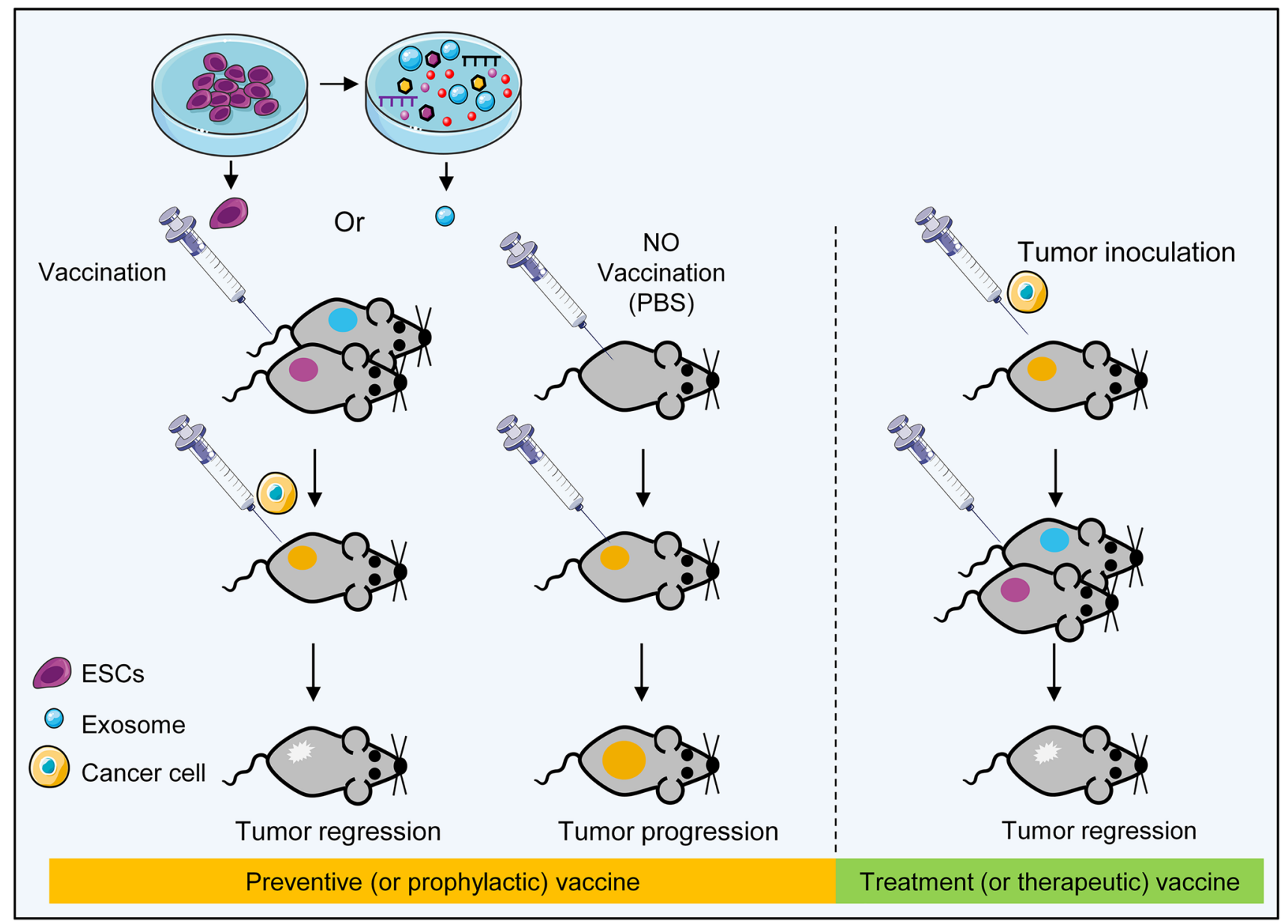

Fig. 6 Pluripotent stem cell (PSC)-derived vaccine. Left: Inactivated PSCs or their extracellular vesicles can induce antitumor immunity that suppresses cancer cell proliferation and prevents tumor devel- opment. Right: PSC-based vaccines can educate the immune system against the cancer and overcome low or no immunogenicity that exist in the tumor microenvironment 
due to chemotherapy or the tumor's immunosuppressive state [161].

Based on this idea, it is suggested that the exposure to conditioned medium, lysate, or mitotically inactivated CSCs or PSCs can activate the immune system and makes immunity against both embryonic and cancer cells [162]. This ability of PSCs can be used by introduction of embryonic materials to cancer cells. The studies show significant reductions in tumor growth and activation of both the humoral and cellular immune systems in response to PSCs. These immunities prevent the growth and proliferation of cancer cells, and an increase in APCs and activation of lymphocytes has been also reported [163]. Interestingly, in these studies, hESCs also produced immune responses in rats, and it can be concluded that the immune function of these cells is species-independent. These vaccines did not show any side effects such as autoimmune reactions [164]. A PSC-derived based vaccine for cancer combined with a histone deacetylase inhibitor (HDACi) could modify the immune suppression microenvironment of the tumor by increasing $\mathrm{CD}^{+}{ }^{+}$or $\mathrm{CD}^{+}$tumor infiltrating lymphocytes and reducing $\mathrm{T}$ regulatory cells. This combined regimen targets CSCs; hence, it could decrease the incidence of metastasis and improve survival rates [165]. Furthermore, vaccination with ESC exosomes, a derivative of these cells in the presence of GM-CSF, resulted in reduced tumor size and immune response [166]. The enhanced targeting ability of ESC exosome carriers combined with chemotherapeutic agents can act highly reactive against tumors [167]. These studies show how ESCs and their secretome act as tumor suppressors and this can be a potential therapeutic approach. The use of exosomes instead of inactivated cells can be a tremendous step towards a cell-free vaccination against cancers (Fig. 6). Overall, these studies showed that with the antigenic similarity to CSCs, PSCs or PSCs-derived secretome therapeutic cancer vaccines can be highly immunogenic against CSCs. As cancer treatment has always been a challenging issue due to the high rate of tumor recurrence and metastasis, PSC-based therapeutic cancer vaccines can theoretically help to overcome the effects of immunosuppression in the tumor microenvironment.

\section{Conclusion and Future Outlook}

Despite multiple therapy attempts, cancer has not been defeated. Tumor cell heterogeneity that causes differences in their biology, metabolism and signaling pathways as well as the effects of the microenvironment on normal cells are challenges that complicate the choice of treatment. Fortunately, recognition of processes such as autophagy has contributed greatly to better identification of cancer cells, and allows for a more effective treatment approach. In order to achieve successful and effective treatment, cancer cells have been targeted based on their recurrence and metastasis. As noted above, there is stemness, pluripotency and the expression of early developmental markers in cancer cells, especially in malignant cancers with poor prognosis. Apart from the assumptions related to the origin of CSCs, the existence of morphological and molecular similarities between cancer cells and early-stage stem cells suggests that early developmental programs are inappropriately activated in cancer; however, the mechanism of this phenomenon is not completely understood. It is assumed that the inappropriate expressions of pluripotency factors and reprogramming of somatic or adult stem cells are the main sources of tumor-like stem cells. There is a defect in developmental biology in these scenarios despite the fact that most developmental biology is observed in embryonic development. Therefore, it seems that a study of embryonic development and its differences and similarities with the developmental processes in cancer can help us to find new therapeutic approaches. Targeting markers and stemness and pluripotency pathways, the use of ESCs as a vaccine, and consideration of embryonic microenvironments in order to regulate developmental phenomena have been investigated to improve cancer treatments.

Acknowledgements Manuscript was written during a doctoral program was approved and funded by Royan Institute for Stem Cell Biology and Technology. This project is also co-funded by Cancer Research Center of Cancer Institute of Iran (Shams cancer charity, Grant No: 37309202-01- 97), and Royan Stem Cell Technology Company.

Author Contributions $\mathrm{MB}, \mathrm{MA}$, and $\mathrm{SN}-\mathrm{H}$ designed the concept of the manuscript. BM, MA, NS, NH, and AG wrote manuscript. MB designed and prepared the figs. $\mathrm{HB}, \mathrm{ME}$, and $\mathrm{SN}-\mathrm{H}$ revised manuscript.

\section{Declarations}

Ethical Approval The content of the manuscript is original and has not been published or accepted somewhere, either in whole or in part. Not part of the manuscript is now under evaluation for publication elsewhere.

Conflict of Interest The authors have no competing interests to declare.

\section{References}

1. WHO. (2020). WHO report on cancer: setting priorities, investing wisely and providing care for all.

2. Vasan, N., Baselga, J., \& Hyman, D. M. (2019). A view on drug resistance in cancer. Nature, 575(7782), 299-309.

3. Brand, N. R., et al. (2019). Delays and Barriers to Cancer Care in Low- and Middle-Income Countries: A Systematic Review. The Oncologist, 24(12), e1371-e1380. 
4. Otto-Meyer, S., et al. (2019). The interplay among psychological distress, the immune system, and brain tumor patient outcomes. Current Opinion in Behavioral Sciences, 28, 44-50.

5. Chapuis, P. H., et al. (2019). Recurrence and cancer-specific death after adjuvant chemotherapy for Stage III colon cancer. Colorectal Disease, 21(2), 164-173.

6. Pan, H., et al. (2017). 20-Year Risks of Breast-Cancer Recurrence after Stopping Endocrine Therapy at 5 Years. New England Journal of Medicine, 377(19), 1836-1846.

7. Reardon, D. A., et al. (2020). Effect of Nivolumab vs Bevacizumab in Patients With Recurrent Glioblastoma: The CheckMate 143 Phase 3 Randomized Clinical Trial. JAMA Oncolology.

8. Rycaj, K., \& Tang, D. G. (2015). Cell-of-Origin of Cancer versus Cancer Stem Cells: Assays and Interpretations. Cancer Research, 75(19), 4003-4011.

9. Akbarzadeh, M., et al. (2019). Current approaches in identification and isolation of cancer stem cells. Journal of Cell Physiology.

10. Ilic, D., \& Ogilvie, C. (2017). Concise Review: Human Embryonic Stem Cells-What Have We Done? What Are We Doing? Where Are We Going? Stem Cells, 35(1), 17-25.

11. Takahashi, K., et al. (2007). Induction of pluripotent stem cells from adult human fibroblasts by defined factors. Cell, 131(5), 861-872.

12. Trounson, A., \& DeWitt, N. D. (2016). Pluripotent stem cells progressing to the clinic. Nature Reviews Molecular Cell Biology, 17(3), 194-200.

13. Yanagimachi, M. D., et al. (2013). Robust and highly-efficient differentiation of functional monocytic cells from human pluripotent stem cells under serum- and feeder cell-free conditions. PLoS One, 8(4), e59243.

14. Zhu, H., et al. (2018). Concise Review: Human Pluripotent Stem Cells to Produce Cell-Based Cancer Immunotherapy. Stem Cells, $36(2), 134-145$.

15. Uenishi, G. I., et al. (2018). NOTCH signaling specifies arterialtype definitive hemogenic endothelium from human pluripotent stem cells. Nature Communications, 9(1), 1828.

16. Postovit, L.-M., et al. (2008). Human embryonic stem cell microenvironment suppresses the tumorigenic phenotype of aggressive cancer cells. Proceedings of the National Academy of Sciences of the United States of America, 105(11), 4329-4334.

17. Yaddanapudi, K., et al. (2012). Vaccination with Embryonic Stem Cells Protects against Lung Cancer: Is a Broad-Spectrum Prophylactic Vaccine against Cancer Possible? PLoS One. 7(7), e42289.

18. Aponte, P. M., \& Caicedo, A. (2017). Stemness in Cancer: Stem Cells, Cancer Stem Cells, and Their Microenvironment. Stem Cells Int, 2017, 5619472.

19. Yadav, A. K., \& Desai, N. S. (2019). Cancer Stem Cells: Acquisition, Characteristics, Therapeutic Implications, Targeting Strategies and Future Prospects. Stem Cell Rev Rep, 15(3), 331-355.

20. Bu, Y., \& Cao, D. (2012). The origin of cancer stem cells. Frontiers in Bioscience (Scholar Edition), 4(3), 819-830.

21. Cooper, M. (2009). Regenerative pathologies: Stem cells, teratomas and theories of cancer. Medicine Studies, 1(1), 55.

22. Ratajczak, M. Z., et al. (2020). The Embryonic Rest Hypothesis of Cancer Development: 150 Years Later, in Trends in Stem Cell Proliferation and Cancer Research, 51-63.

23. Hohenstein, P., Pritchard-Jones, K., \& Charlton, J. (2015). The yin and yang of kidney development and Wilms' tumors. Genes \& Development, 29(5), 467-482.

24. Shin, D. M., et al. (2010). Molecular signature of adult bone marrow-purified very small embryonic-like stem cells supports their developmental epiblast/germ line origin. Leukemia, 24(8), $1450-1461$
25. Ratajczak, M. Z., Ratajczak, J., \& Kucia, M. (2019). Very Small Embryonic-Like Stem Cells (VSELs). Circulation Research, 124(2), 208-210.

26. Ratajczak, M. Z., et al. (2018). Cancer from the perspective of stem cells and misappropriated tissue regeneration mechanisms. Leukemia, 32(12), 2519-2526.

27. Zuba-Surma, E. K., et al. (2009). Very small embryonic-like stem cells in adult tissues-potential implications for aging. Mechanisms of Ageing and Development, 130(1-2), 58-66.

28. Virant-Klun, I., et al. (2019). Similar population of CD133+ and DDX4+ VSEL-like stem cells sorted from human embryonic stem cell, ovarian, and ovarian Cancer ascites cell cultures: The real embryonic stem cells? Cells, 8(7), 706.

29. Ratajczak, M. Z., et al. (2011). Identification of very small embryonic/epiblast-like stem cells (VSELs) circulating in peripheral blood during organ/tissue injuries. Methods in Cell Biology, 103, 31-54.

30. Bhartiya, D. (2017). Pluripotent Stem Cells in Adult Tissues: Struggling To Be Acknowledged Over Two Decades. Stem Cell Rev Rep, 13(6), 713-724.

31. Ratajczak, M. Z., et al. (2014). Very small embryonic-like stem cells (VSELs) represent a real challenge in stem cell biology: Recent pros and cons in the midst of a lively debate. Leukemia, 28(3), 473-484.

32. Ratajczak, M. Z., et al. (2011). RasGrf1: Genomic imprinting, VSELs, and aging. Aging (Albany NY), 3(7), 692.

33. Shin, D. M., et al. (2009). Novel epigenetic mechanisms that control pluripotency and quiescence of adult bone marrow-derived Oct4(+) very small embryonic-like stem cells. Leukemia, 23(11), 2042-2051.

34. Peters, J. (2014). The role of genomic imprinting in biology and disease: An expanding view. Nature Reviews Genetics, 15(8), 517-530.

35. Singh, P., Lee, D.-H., \& Szabó, P. E. (2012). More than insulator: Multiple roles of CTCF at the H19-Igf2 imprinted domain. Frontiers in genetics, 3, 214.

36. Kaushik, A., Anand, S., \& Bhartiya, D. (2020). Altered biology of testicular VSELs and SSCs by neonatal endocrine disruption results in defective spermatogenesis, reduced fertility and tumor initiation in adult mice. Stem Cell Reviews and Reports, 16(5), 893-908.

37. Kaushik, A., \& Bhartiya, D. (2018). Pluripotent very small embryonic-like stem cells in adult testes-an alternate premise to explain testicular germ cell tumors. Stem cell reviews and reports, 14(6), 793-800.

38. Virant-Klun, I., \& Stimpfel, M. (2016). Novel population of small tumour-initiating stem cells in the ovaries of women with borderline ovarian cancer. Scientific reports, 6(1), 1-23.

39. Virant-Klun, I., Kenda-Suster, N., \& Smrkolj, S. (2016). Small putative NANOG, SOX2, and SSEA-4-positive stem cells resembling very small embryonic-like stem cells in sections of ovarian tissue in patients with ovarian cancer. Journal of ovarian research, 9(1), 1-15.

40. Beumer, J., \& Clevers, H. (2016). Regulation and plasticity of intestinal stem cells during homeostasis and regeneration. Development, 143(20), 3639-3649.

41. Colacino, J. A., et al. (2018). Heterogeneity of Human Breast Stem and Progenitor Cells as Revealed by Transcriptional Profiling. Stem Cell Reports, 10(5), 1596-1609.

42. Kim, C. F., et al. (2005). Identification of bronchioalveolar stem cells in normal lung and lung cancer. Cell, 121(6), 823-835.

43. Ng, A., \& Barker, N. (2015). Ovary and fimbrial stem cells: Biology, niche and cancer origins. Nature Reviews Molecular Cell Biology, 16(10), 625-638.

44. Wang, X., et al. (2009). A luminal epithelial stem cell that is a cell of origin for prostate cancer. Nature, 461(7263), 495-500. 
45. Batlle, E., \& Clevers, H. (2017). Cancer stem cells revisited. Nature medicine, 23(10), 1124.

46. Visvader, J. E., \& Clevers, H. (2016). Tissue-specific designs of stem cell hierarchies. Nature Cell Biology, 18(4), 349-355.

47. Rodgers, J. T., et al. (2014). mTORC1 controls the adaptive transition of quiescent stem cells from G0 to G(Alert). Nature, 510(7505), 393-396.

48. Chen, X., He, Y., \& Lu, F. (2018). Autophagy in Stem Cell Biology: A Perspective on Stem Cell Self-Renewal and Differentiation. Stem Cells Int, 2018, 9131397.

49. Montagnani, S., et al. (2016). Adult Stem Cells in Tissue Maintenance and Regeneration. Stem Cells Int, 2016, 7362879.

50. Blokzijl, F., et al. (2016). Tissue-specific mutation accumulation in human adult stem cells during life. Nature, 538(7624), 260-264.

51. Covey, M. V., et al. (2012). REST regulates the pool size of the different neural lineages by restricting the generation of neurons and oligodendrocytes from neural stem/progenitor cells. Development, 139(16), 2878-2890.

52. Adams, P. D., Jasper, H., \& Rudolph, K. L. (2015). AgingInduced Stem Cell Mutations as Drivers for Disease and Cancer. Cell Stem Cell, 16(6), 601-612.

53. Bjerkvig, R., et al. (2005). The origin of the cancer stem cell: Current controversies and new insights. Nature Reviews Cancer, 5(11), 899-904.

54. Biswas, S., et al. (2015). Microenvironmental control of stem cell fate in intestinal homeostasis and disease. The Journal of Pathology, 237(2), 135-145.

55. Redondo, P. A., et al. (2017). Elements of the niche for adult stem cell expansion. J Tissue Eng, 8, 2041731417725464.

56. Sharif, T., et al. (2017). Autophagic homeostasis is required for the pluripotency of cancer stem cells. Autophagy, 13(2), 264-284.

57. Nazio, F., et al. (2019). Autophagy and cancer stem cells: Molecular mechanisms and therapeutic applications. Cell Death and Differentiation, 26(4), 690-702.

58. Cole, A. J., et al. (2020). An evolving paradigm of cancer stem cell hierarchies: Therapeutic implications. Theranostics, 10(7), 3083-3098.

59. Bigas, A., \& Espinosa, L. (2016). Notch Signaling in Cell-Cell Communication Pathways. Current Stem Cell Reports, 2(4), 349-355.

60. Takebe, N., et al. (2015). Targeting Notch, Hedgehog, and Wnt pathways in cancer stem cells: Clinical update. Nature Reviews. Clinical Oncology, 12(8), 445-464.

61. Uchida, N., et al. (2000). Direct isolation of human central nervous system stem cells. Proceedings of the national academy of sciences, 97(26), 14720-14725.

62. Williams, K., et al. (2013). CD44 integrates signaling in normal stem cell, cancer stem cell and (pre)metastatic niches. Experimental Biology and Medicine (Maywood, N.J.), 238(3), 324-338.

63. Barzegar Behrooz, A., Syahir, A., Ahmad S. (2019). CD133: beyond a cancer stem cell biomarker. Journal of Drug Targeting, 27(3), 257-269.

64. Prager, B. C., et al. (2019). Cancer Stem Cells: The Architects of the Tumor Ecosystem. Cell Stem Cell, 24(1), 41-53.

65. Krebsbach, P. H., \& Villa-Diaz, L. G. (2017). The Role of Integrin alpha6 (CD49f) in Stem Cells: More than a Conserved Biomarker. Stem Cells Dev, 26(15), 1090-1099.

66. Martin, T. A., \& Jiang, W. G. (2014). Evaluation of the expression of stem cell markers in human breast cancer reveals a correlation with clinical progression and metastatic disease in ductal carcinoma. Oncology Reports, 31(1), 262-272.
67. Zhu, L., et al. (2009). Prominin 1 marks intestinal stem cells that are susceptible to neoplastic transformation. Nature, 457(7229), 603-607.

68. Takahashi, K., \& Yamanaka, S. (2006). Induction of pluripotent stem cells from mouse embryonic and adult fibroblast cultures by defined factors. Cell, 126(4), 663-676.

69. Villodre, E. S., et al. (2019). Silencing of the transcription factors Oct4, Sox2, Klf4, c-Myc or Nanog has different effect on teratoma growth. Biochemical and Biophysical Research Communications, 517(2), 324-329.

70. Friedmann-Morvinski, D., \& Verma, I. M. (2014). Dedifferentiation and reprogramming: Origins of cancer stem cells. EMBO Reports, 15(3), 244-253.

71. Chaffer, C. L., et al. (2011). Normal and neoplastic nonstem cells can spontaneously convert to a stem-like state. Proc Natl Acad Sci U S A, 108(19), 7950-7955.

72. Tata, P. R., et al. (2013). Dedifferentiation of committed epithelial cells into stem cells in vivo. Nature, 503(7475), 218-223.

73. Schwitalla, S., et al. (2013). Intestinal tumorigenesis initiated by dedifferentiation and acquisition of stem-cell-like properties. Cell, 152(1-2), 25-38.

74. Guerra, C., et al. (2007). Chronic pancreatitis is essential for induction of pancreatic ductal adenocarcinoma by K-Ras oncogenes in adult mice. Cancer Cell, 11(3), 291-302.

75. Gidekel Friedlander, S. Y., et al. (2009). Context-dependent transformation of adult pancreatic cells by oncogenic K-Ras. Cancer Cell, 16(5), 379-389.

76. Hwang, J. H., et al. (2020). A mutant KRAS-induced factor REG4 promotes cancer stem cell properties via Wnt/beta-catenin signaling. International Journal of Cancer, 146(10), 2877-2890.

77. Vermeulen, L., et al. (2010). Wnt activity defines colon cancer stem cells and is regulated by the microenvironment. Nature Cell Biology, 12(5), 468-476.

78. Landsberg, J., et al. (2012). Melanomas resist T-cell therapy through inflammation-induced reversible dedifferentiation. Nature, 490(7420), 412-416.

79. Hadjimichael, C., et al. (2015). Common stemness regulators of embryonic and cancer stem cells. World J Stem Cells, 7(9), $1150-1184$.

80. Kim, J., \& Orkin, S. H. (2011). Embryonic stem cell-specific signatures in cancer: Insights into genomic regulatory networks and implications for medicine. Genome medicine, 3(11), 1-8.

81. Jang, H., et al. (2015). Metabolism in embryonic and cancer stemness. Archives of Pharmacal Research, 38(3), 381-388.

82. Muller, M., et al. (2016). The role of pluripotency factors to drive stemness in gastrointestinal cancer. Stem Cell Res, 16(2), 349-357.

83. Cho, Y., et al. (2018). Post-translational modification of OCT4 in breast cancer tumorigenesis. Cell Death and Differentiation, 25(10), 1781-1795.

84. Rasti, A., et al. (2018). Co-expression of Cancer Stem Cell Markers OCT4 and NANOG Predicts Poor Prognosis in Renal Cell Carcinomas. Science and Reports, 8(1), 11739.

85. Lu, Y., et al. (2013). Knockdown of Oct4 and Nanog expression inhibits the stemness of pancreatic cancer cells. Cancer Letters, 340(1), 113-123.

86. Zbinden, M., et al. (2010). NANOG regulates glioma stem cells and is essential in vivo acting in a cross-functional network with GLI1 and p53. EMBO Journal, 29(15), 2659-2674.

87. Lee, T. K., et al. (2011). CD24(+) liver tumor-initiating cells drive self-renewal and tumor initiation through STAT3-mediated NANOG regulation. Cell Stem Cell, 9(1), 50-63.

88. Ibrahim, E. E., et al. (2012). Embryonic NANOG activity defines colorectal cancer stem cells and modulates through AP1- and TCF-dependent mechanisms. Stem Cells, 30(10), 2076-2087. 
89. Wang, X., et al. (2019). AMPK Promotes SPOP-Mediated NANOG Degradation to Regulate Prostate Cancer Cell Stemness. Developmental Cell, 48(3), 345-360 e7.

90. Wang, Z., et al. (2019). AKT drives SOX2 overexpression and cancer cell stemness in esophageal cancer by protecting SOX2 from UBR5-mediated degradation. Oncogene, 38(26), $5250-5264$

91. Bora-Singhal, N., et al. (2020). Novel HDAC11 inhibitors suppress lung adenocarcinoma stem cell self-renewal and overcome drug resistance by suppressing Sox2. Science and Reports, 10(1), 4722 .

92. Yin, Y., et al. (2019). The FBXW2-MSX2-SOX2 axis regulates stem cell property and drug resistance of cancer cells. Proc Natl Acad Sci U S A, 116(41), 20528-20538.

93. Tseng, W. C., et al. (2016). Kruppel-like factor 4 is a novel prognostic predictor for urothelial carcinoma of bladder and it regulates TWIST1-mediated epithelial-mesenchymal transition. Urologic Oncology, 34(11), 485 e15-485 e24.

94. Qi, X. T., et al. (2019). KLF4 functions as an oncogene in promoting cancer stem cell-like characteristics in osteosarcoma cells. Acta Pharmacologica Sinica, 40(4), 546-555.

95. Ma, Y., et al. (2017). KLF4 inhibits colorectal cancer cell proliferation dependent on NDRG2 signaling. Oncology Reports, 38(2), 975-984.

96. Shum, C. K., et al. (2013). Kruppel-like factor 4 (KLF4) suppresses neuroblastoma cell growth and determines non-tumorigenic lineage differentiation. Oncogene, 32(35), 4086-4099.

97. Li, X., et al. (2017). Klf4 reduces stemness phenotype, triggers mesenchymal-epithelial transition (MET)-like molecular changes, and prevents tumor progression in nasopharygeal carcinoma. Oncotarget, 8(55), 93924-93941.

98. Tapia, N., \& Scholer, H. R. (2010). p53 connects tumorigenesis and reprogramming to pluripotency. Journal of Experimental Medicine, 207(10), 2045-2048.

99. Hoffmeyer, K., et al. (2012). Wnt/beta-catenin signaling regulates telomerase in stem cells and cancer cells. Science, 336(6088), $1549-1554$.

100. Dang, C. V. (2012). MYC on the path to cancer. Cell, 149(1), $22-35$.

101. Lin, C. Y., et al. (2012). Transcriptional amplification in tumor cells with elevated c-Myc. Cell, 151(1), 56-67.

102. Dreesen, O., \& Brivanlou, A. H. (2007). Signaling pathways in cancer and embryonic stem cells. Stem Cell Rev, 3(1), 7-17.

103. Noggle, S. A., Weiler, D., \& Condie, B. G. (2006). Notch signaling is inactive but inducible in human embryonic stem cells. Stem Cells, 24(7), 1646-1653.

104. Lowell, S., et al. (2006). Notch promotes neural lineage entry by pluripotent embryonic stem cells. PLoS Biology, 4(5), e121.

105. Liu, W., et al. (2013). Blockage of Notch signaling inhibits the migration and proliferation of retinal pigment epithelial cells. Scientific World Journal, 178708.

106. Vujovic, F., Hunter, N., \& Farahani, R. M. (2019). Notch pathway: A bistable inducer of biological noise? Cell Communication and Signaling: CCS, 17(1), 133.

107. Asnaghi, L., et al. (2012). Notch signaling promotes growth and invasion in uveal melanoma. Clinical Cancer Research, 18(3), 654-665.

108. Hallahan, A. R., et al. (2004). The SmoA1 mouse model reveals that notch signaling is critical for the growth and survival of sonic hedgehog-induced medulloblastomas. Cancer research, 64(21), 7794-7800.

109. McAuliffe, S. M., et al. (2012). Targeting Notch, a key pathway for ovarian cancer stem cells, sensitizes tumors to platinum therapy. Proc Natl Acad Sci U S A, 109(43), E2939-E2948.
110. Stylianou, S., Clarke, R. B., \& Brennan, K. (2006). Aberrant activation of notch signaling in human breast cancer. Cancer Research, 66(3), 1517-1525.

111. Nowell, C. S., \& Radtke, F. (2017). Notch as a tumour suppressor. Nature Reviews Cancer, 17(3), 145-159.

112. Loh, K. M., van Amerongen, R., \& Nusse, R. (2016). Generating Cellular Diversity and Spatial Form: Wnt Signaling and the Evolution of Multicellular Animals. Developmental Cell, 38(6), 643-655.

113. Xu, Z., et al. (2016). Wnt/beta-catenin signaling promotes selfrenewal and inhibits the primed state transition in naive human embryonic stem cells. Proc Natl Acad Sci U S A, 113(42), E6382-E6390.

114. Duchartre, Y., Kim, Y. M., \& Kahn, M. (2016). The Wnt signaling pathway in cancer. Critical Reviews in Oncology Hematology, 99, 141-149.

115. Polakis, P. (2012). Wnt signaling in cancer. Cold Spring Harbor Perspectives Biology, 4(5).

116. Zhan, T., Rindtorff, N., \& Boutros, M. (2017). Wnt signaling in cancer. Oncogene, 36(11), 1461-1473.

117. Borah, A., et al. (2015). Targeting self-renewal pathways in cancer stem cells: clinical implications for cancer therapy. Oncogenesis, 4, e177.

118. Krausova, M., \& Korinek, V. (2014). Wnt signaling in adult intestinal stem cells and cancer. Cellular Signalling, 26(3), 570-579.

119. Xue, G., et al. (2016). Wnt/beta-catenin signaling in melanoma: Preclinical rationale and novel therapeutic insights. Cancer Treatment Reviews, 49, 1-12.

120. Sun, J., et al. (2019). Low-level shear stress induces differentiation of liver cancer stem cells via the Wnt/beta-catenin signalling pathway. Experimental Cell Research, 375(1), 90-96.

121. Zhang, K., et al. (2017). WNT/beta-Catenin Directs SelfRenewal Symmetric Cell Division of hTERT(high) Prostate Cancer Stem Cells. Cancer Research, 77(9), 2534-2547.

122. Pohl, S. G., et al. (2017). Wnt signaling in triple-negative breast cancer. Oncogenesis, 6(4), e310.

123. Oren, O., \& Smith, B. D. (2017). Eliminating Cancer Stem Cells by Targeting Embryonic Signaling Pathways. Stem Cell Rev Rep, 13(1), 17-23.

124. Katoh, M. (2011). Network of WNT and other regulatory signaling cascades in pluripotent stem cells and cancer stem cells. Current pharmaceutical biotechnology, 12(2), 160-170.

125. Sari, I. N., et al. (2018). Hedgehog Signaling in Cancer: A Prospective Therapeutic Target for Eradicating Cancer Stem Cells. Cells, 7(11).

126. Li, Q. R., et al. (2019). Novel-smoothened inhibitors for therapeutic targeting of naive and drug-resistant hedgehog pathwaydriven cancers. Acta Pharmacologica Sinica, 40(2), 257-267.

127. Brewer, B. G., et al. (2009). Embryonic vaccines against cancer: An early history. Experimental and Molecular Pathology, 86(3), 192-197.

128. Jiang, X. P., et al. (2000). Vaccination with a mixed vaccine of autogenous and allogeneic breast cancer cells and tumor associated antigens CA15-3, CEA and CA125-results in immune and clinical responses in breast cancer patients. Cancer biotherapy \& radiopharmaceuticals, 15(5), 495-505.

129. Malatesta, M., et al. (2000). Prostate-specific antigen synthesis and secretion by human placenta: A physiological kallikrein source during pregnancy. The Journal of Clinical Endocrinology \& Metabolism, 85(1), 317-321.

130. Gjerstorff, M. F., Andersen, M. H., \& Ditzel, H. J. (2015). Oncogenic cancer/testis antigens: Prime candidates for immunotherapy. Oncotarget, 6(18), 15772. 
131. Skinner, J., \& Whitehead, R. (1981). Tumor-associated antigens in polyps and carcinoma of the human large bowel. Cancer, 47(6), 1241-1245.

132. Han, S. N., et al. (2012). Physiologic variations of serum tumor markers in gynecological malignancies during pregnancy: A systematic review. BMC medicine, 10(1), 86.

133. Beaman, K. D., et al. (2016). Pregnancy is a model for tumors, not transplantation. American Journal of Reproductive Immunology, 76(1), 3-7.

134. Hepner, A., et al. (2019). Cancer During Pregnancy: The Oncologist Overview. World J Oncol, 10(1), 28-34.

135. Ouyang, X., Telli, M. L., \& Wu, J. C. (2019). Induced Pluripotent Stem Cell-Based Cancer Vaccines. Frontiers in Immunology, 10, 1510.

136. Camara, D. A., et al. (2016). Advances and Challenges on Cancer Cells Reprogramming Using Induced Pluripotent Stem Cells Technologies. Journal of Cancer, 7(15), 2296-2303.

137. Bindhya, S., et al. (2019). Induced pluripotent stem cells: A new strategy to model human cancer. International Journal of Biochemistry \& Cell Biology, 107, 62-68.

138. Lee, D. F., et al. (2015). Modeling familial cancer with induced pluripotent stem cells. Cell, 161(2), 240-254.

139. Kotini, A. G., et al. (2015). Functional analysis of a chromosomal deletion associated with myelodysplastic syndromes using isogenic human induced pluripotent stem cells. Nature Biotechnology, 33(6), 646-655.

140. Kim, J., et al. (2013). An iPSC line from human pancreatic ductal adenocarcinoma undergoes early to invasive stages of pancreatic cancer progression. Cell Reports, 3(6), 2088-2099.

141. Sommer, C. A., et al. (2018). Modeling APC mutagenesis and familial adenomatous polyposis using human iPS cells. PLoS One, 13(7), e0200657.

142. Chen, L., et al. (2012). A model of cancer stem cells derived from mouse induced pluripotent stem cells. PLoS One, 7(4), e33544.

143. Yan, T., et al. (2014). Characterization of cancer stem-like cells derived from mouse induced pluripotent stem cells transformed by tumor-derived extracellular vesicles. Journal of Cancer, 5(7), 572-584.

144. Prieto-Vila, M., et al. (2016). iPSC-derived cancer stem cells provide a model of tumor vasculature. American Journal of Cancer Research, 6(9), 1906-1921.

145. Xu, N., et al. (2018). Induction of cells with prostate cancer stem-like properties from mouse induced pluripotent stem cells via conditioned medium. American journal of cancer research, $8(8), 1624$.

146. Papapetrou, E. P. (2016). Patient-derived induced pluripotent stem cells in cancer research and precision oncology. Nature medicine, 22(12), 1392-1401.

147. Postovit, L. M., et al. (2006). A three-dimensional model to study the epigenetic effects induced by the microenvironment of human embryonic stem cells. Stem Cells, 24(3), 501-505.

148. Giuffrida, D., et al. (2009). Human embryonic stem cells secrete soluble factors that inhibit cancer cell growth. Cell Proliferation, 42(6), 788-798.

149. Raof, N. A., et al. (2011). Bioengineering embryonic stem cell microenvironments for exploring inhibitory effects on metastatic breast cancer cells. Biomaterials, 32(17), 4130-4139.

150. Zhou, S., et al. (2017). Reprogramming Malignant Cancer Cells toward a Benign Phenotype following Exposure to Human Embryonic Stem Cell Microenvironment. PLoS One, 12(1), e0169899.

151. Lan, G., et al. (2019). Notch pathway is involved in the suppression of colorectal cancer by embryonic stem cell microenvironment. Oncotargets and Therapy, 12, 2869-2878.
152. Raof, N. A., Mooney, B. M., \& Xie, Y. (2011). Bioengineering embryonic stem cell microenvironments for the study of breast cancer. International Journal of Molecular Sciences, 12(11), 7662-7691.

153. Costa, F. F., et al. (2009). Epigenetically reprogramming metastatic tumor cells with an embryonic microenvironment. Epigenomics, 1(2), 387-398.

154. Kulesa, P. M., et al. (2006). Reprogramming metastatic melanoma cells to assume a neural crest cell-like phenotype in an embryonic microenvironment. Proceedings of the National Academy of Sciences, 103(10), 3752-3757.

155. Illmensee, K., \& Mintz, B. (1976). Totipotency and normal differentiation of single teratocarcinoma cells cloned by injection into blastocysts. Proceedings of the National Academy of Sciences, 73(2), 549-553.

156. Li, Y., et al. (2009). Vaccination with human pluripotent stem cells generates a broad spectrum of immunological and clinical responses against colon cancer. Stem Cells, 27(12), 3103-3111.

157. Zhang, Z., et al. (2013). Vaccination with embryonic stem cells generates effective antitumor immunity against ovarian cancer. International Journal of Molecular Medicine, 31(1), 147-153.

158. Wang, Q. T., et al. (2020). Tumor-associated antigen-based personalized dendritic cell vaccine in solid tumor patients. Cancer Immunology, Immunotherapy, 1-13.

159. Avigan, D., \& Rosenblatt, J. (2018). Vaccine therapy in hematologic malignancies. Blood, 131(24), 2640-2650.

160. Wang, Y., et al. (2020). Dendritic cell biology and its role in tumor immunotherapy. Journal of Hematology \& Oncology, 13(1), 1-18.

161. Rami, F., H. (2016). Mollainezhad, and M. Salehi, Induced pluripotent stem cell as a new source for cancer immunotherapy. Genetics Research International.

162. Dhodapkar, M. V. (2010). Immunity to stemness genes in human cancer. Current Opinion in Immunology, 22(2), 245-250.

163. Kooreman, N. G., et al. (2020). Autologous iPSC-Based Vaccines Elicit Anti-tumor Responses In Vivo. Cell Stem Cell, 22(4), 501-513 e 7.

164. Zhang, Z. J., et al. (2012). Human embryonic stem cells-a potential vaccine for ovarian cancer. Asian Pacific Journal of Cancer Prevention, 13(9), 4295-4300.

165. Heront-Kishi, M., et al. (2020). Pharmacologically modified pluripotent stem cell-based cancer vaccines with anti-metastatic potential, bioRxiv.

166. Yaddanapudi, K., et al. (2019). Exosomes from GM-CSF expressing embryonic stem cells are an effective prophylactic vaccine for cancer prevention. Oncoimmunology, 8(3), 1561119.

167. Zhu, Q., et al. (2019). Embryonic Stem Cells-Derived Exosomes Endowed with Targeting Properties as Chemotherapeutics Delivery Vehicles for Glioblastoma Therapy. Adv Sci (Weinh), 6(6), 1801899.

168. Gwak, J. M., et al. (2017). Expression of embryonal stem cell transcription factors in breast cancer: Oct4 as an indicator for poor clinical outcome and tamoxifen resistance. Oncotarget, $8(22), 36305$

169. Cheng, C. C., et al. (2018). Stat3/Oct-4/c-Myc signal circuit for regulating stemness-mediated doxorubicin resistance of triplenegative breast cancer cells and inhibitory effects of WP1066. International journal of oncology, 53(1), 339-348.

170. Ghanei, Z., et al. (2020). Isolation and characterization of breast cancer stem cell-like phenotype by Oct4 promoter-mediated activity. Journal of cellular physiology, 235(11), 7840-7848.

171. Shankar, S., et al. (2011) Resveratrol inhibits pancreatic cancer stem cell characteristics in human and Kras G12D transgenic mice by inhibiting pluripotency maintaining factors and epithelial-mesenchymal transition. PloS one, 6(1), e16530. 
172. Jia, Q., et al. (2013). Positive correlation of Oct4 and ABCG2 to chemotherapeutic resistance in CD90+ CD133+ liver cancer stem cells. Cellular reprogramming, 15(2), 143-150.

173. Boumahdi, S., et al. (2014). SOX2 controls tumour initiation and cancer stem-cell functions in squamous-cell carcinoma. Nature, 511(7508), 246-250.

174. Xiang, R., et al. (2011). Downregulation of transcription factor SOX2 in cancer stem cells suppresses growth and metastasis of lung cancer. British journal of cancer, 104(9), 1410-1417.

175. Gangemi, R. M. R., et al. (2009). SOX2 silencing in glioblastoma tumor-initiating cells causes stop of proliferation and loss of tumorigenicity. Stem cells, 27(1), 40-48.

176. Lundberg, I. V., et al. (2016). SOX2 expression is associated with a cancer stem cell state and down-regulation of CDX2 in colorectal cancer. BMC Cancer, 16(1), 1-11.

177. Zhang, P., et al. (2016). MicroRNA-10b regulates epithelialmesenchymal transition by modulating KLF4/Notch1/E-cadherin in cisplatin-resistant nasopharyngeal carcinoma cells. American journal of cancer research, 6(2), 141.

178. Shan, J., et al. (2012). Nanog regulates self-renewal of cancer stem cells through the insulin-like growth factor pathway in human hepatocellular carcinoma. Hepatology, 56(3), 1004-1014.

179. Jeter, C. R., et al. (2011). NANOG promotes cancer stem cell characteristics and prostate cancer resistance to androgen deprivation. Oncogene, 30(36), 3833-3845.

180. Yong, X., et al. (2016). Helicobacter pylori upregulates Nanog and Oct 4 via Wnt/ $\beta$-catenin signaling pathway to promote cancer stem cell-like properties in human gastric cancer. Cancer letters, 374(2), 292-303.

181. Avila, J. L., \& Kissil, J. L. (2013). Notch signaling in pancreatic cancer: Oncogene or tumor suppressor? Trends in molecular medicine, 19(5), 320-327.

182. Wang, Y., et al. (2009). A side population of cells from a human pancreatic carcinoma cell line harbors cancer stem cell characteristics. Neoplasma, 56(5), 371.

183. Andrew, R., Hallahan, et al. (2004). The SmoA1 Mouse Model Reveals That Notch Signaling Is Critical for the Growth and Survival of Sonic Hedgehog-Induced Medulloblastomas. Cancer Research, 64,7794-7800.

184. Wang, L., et al. (2020). Inhibition of Notch pathway enhances the anti-tumor effect of docetaxel in prostate cancer stem-like cells. Stem Cell Research \& Therapy, 11(1), 1-8.

185. Huang, Q., et al. (2019). The carcinogenic role of the notch signaling pathway in the development of hepatocellular carcinoma. Journal of Cancer, 10(6), 1570.

186. Westhoff, B., et al. (2009). Alterations of the Notch pathway in lung cancer. Proceedings of the National Academy of Sciences, 106(52), 22293-22298.
187. Nguyen, B. C., et al. (2006). Cross-regulation between Notch and p63 in keratinocyte commitment to differentiation. Genes \& development, 20(8), 1028-1042.

188. Koch, U., \& Radtke, F. (2007). Notch and cancer: A doubleedged sword. Cellular and molecular life sciences, 64(21), 2746-2762.

189. Zhang, M., et al. (2016). Does Notch play a tumor suppressor role across diverse squamous cell carcinomas? Cancer medicine, 5(8), 2048-2060.

190. Shah, P. A., et al. (2020). NOTCH1 Signaling in Head and Neck Squamous Cell Carcinoma. Cells, 9(12), 2677.

191. Sriuranpong, V., et al. (2001). Notch signaling induces cell cycle arrest in small cell lung cancer cells. Cancer research, 61(7), 3200-3205.

192. Augert, A., et al. (2019). Targeting NOTCH activation in small cell lung cancer through LSD1 inhibition. Science signaling, 12(567).

193. Teglund, S., Toftgård, R. (2010). Hedgehog beyond medulloblastoma and basal cell carcinoma. Biochimica et Biophysica Acta (BBA)-Reviews on Cancer, 1805(2), 181-208.

194. Bonifas, J. M., et al. (2001). Activation of expression of hedgehog target genes in basal cell carcinomas. Journal of investigative dermatology, 116(5), 739-742.

195. Berman, D. M., et al. (2002). Medulloblastoma growth inhibition by hedgehog pathway blockade. Science, 297(5586), 1559-1561.

196. Varnat, F., et al. (2009). Human colon cancer epithelial cells harbour active HEDGEHOG-GLI signalling that is essential for tumour growth, recurrence, metastasis and stem cell survival and expansion. EMBO molecular medicine, 1(6-7), 338-351.

197. Liu, S., et al. (2006). Hedgehog signaling and Bmi-1 regulate self-renewal of normal and malignant human mammary stem cells. Cancer research, 66(12), 6063-6071.

198. Zhao, C., et al. (2009). Hedgehog signalling is essential for maintenance of cancer stem cells in myeloid leukaemia. Nature, 458(7239), 776-779.

199. Burns, M. A., et al. (2018). Hedgehog pathway mutations drive oncogenic transformation in high-risk T-cell acute lymphoblastic leukemia. Leukemia, 32(10), 2126-2137.

200. di Magliano, M. P., et al. (2006). Hedgehog/Ras interactions regulate early stages of pancreatic cancer. Genes \& development, 20(22), 3161-3173.

Publisher's Note Springer Nature remains neutral with regard to jurisdictional claims in published maps and institutional affiliations. 